\title{
Assessment of robust optimization for design of rotorcraft airfoils in forward flight
}

\author{
F. Fusi ${ }^{\mathrm{a}}$, P.M. Congedo ${ }^{\mathrm{b}}$, A. Guardone ${ }^{\mathrm{a}}$, G. Quaranta ${ }^{\mathrm{a}}$ \\ ${ }^{a}$ Department of Aerospace Science and Technology, Politecnico di Milano, Via La Masa \\ 34, 20156 Milano, Italy \\ ${ }^{b}$ Team CARDAMOM, INRIA Bordeaux Sud-Ouest, Talence, 33405 Cedex, France
}

\begin{abstract}
The paper compares the deterministic and robust approaches to improve the aerodynamic design of helicopter airfoils. The two formulations are different due to the characteristics of each approach. In the deterministic case, the objective of optimization is the minimization of drag while maintaining a level of lift that guarantees satisfaction of trimming condition. In the case of robust design, a range of angles of attack and not a single trim condition is considered. Thus, the robust optimization takes the lift-to-drag ratio as a measure of the performance of the airfoil, imposing at the same time inequality constraint on the lift coefficient to guarantee a sufficient level of lift, and then checking after optimization that the trimming condition can be satisfied. The two approaches are compared showing pros and cons of the robust framework. In general, the robust approach shows the capability to reach the same mean performance of the deterministic one, but with a lower degradation of performances in off-design situations considered through the uncertainty. On the other side, the difficulties in imposing the lift trim condition for the robust formulation may lead to results of limited use.

Keywords: Airfoil design, unsteady aerodynamics, uncertainty quantification, robust optimization.
\end{abstract}




\section{Introduction}

Forward flight is one of the most peculiar and complex flow condition for rotorcraft blades. The flow encountered by the blade in such a condition is periodically changing over the azimuth angle and the extremes of this cycle are very different from each other [1], see Figure 1 . On the advancing side, the rotor blade encounters transonic flow and low values of the angle of attack. On the retreating side, the angle of attack is typically high and the blade section Mach number is lower, typically in the subsonic range or even close to the incompressible limit. For this reason, aerodynamic design should take into account the variability of the flow encountered by the blade, because it is highly possible that an improvement in some conditions may cause a deterioration of performance in others [2].

The unsteady flow encountered on a rotorcraft blade affect the prediction of both local aerodynamics along each blade section and the overall performance. A proper simulation of the complex rotor blade environment requires considering simultaneous speed and angle of attack fluctuations of the incoming airflow, compressibility effects, three-dimensionality of the flow, accurate viscous separation modelling and coupling of the aerodynamic loads with the blade dynamics and aeroelasticity. The accurate prediction of rotor loads requires the definition of detailed computational fluid dynamics (CFD) and computational structural dynamics (CSD) models coupled through appropriate procedures [3]. Even with such models the correlation with experiments is often not so good. In a fundamental work, Bhagwat et al. [4] showed that three-dimensional (3-D) CFD/CSD simulation of complex maneuvered flight conditions may improve the quality of the predicted loads. However, very refined grids are necessary together with computationally expensive turbulence models, as shown also in Ref. [5]. 
Several authors employed 3-D models to perform the blade geometry optimization in hover. Le Pape and Baumier [6] used the 3-D models to optimize twist, chord, sweep and anhedral distributions, while Sun and Lee [7] in the same flow condition optimized also airfoil shape. Many other authors used 3-D detailed models to perform the rotor optimization in forward flight too [8-11]. However, the computational cost is very high, and consequently it is hard to conjugate the complexity with the necessity to obtain good performances under a range of operating conditions, including off-design situations, as suggested for any aerodynamic optimization in Ref. [12]. Consequently, many design approaches regarding aerodynamics of blades have tackled the problem by separating the influence of these combined effects. In this framework, it still makes sense to start from the optimization of the blade airfoils at different radii, and then to work on the optimization of other geometrical elements. There is a consensus on the correlation between the lift-to-drag $L / D$ ratio of the airfoil and the $L / D$ ratio of the rotor $[13,14]$, so this could be taken as performance objective together with the maximization of lift coefficient in the high angle-of-attack flow conditions.

However, even in the case of optimization of airfoils, the selection of operating conditions is not an easy task. In fact, each airfoil during each turn is subject to a range of angles of attack and Mach numbers due to the combination of the rotational velocity with flight speed (see Figure 2). This is presented in Fig. 1 where the operating conditions of a blade element are shown for different blade section radial positions. It is clear that both the local angle of attack $\alpha$ and the freestream Mach number $M$ undergo a periodic variation over the rotation period.

To take into account the variation of flow conditions over the azimuth angle, a fully unsteady optimization may be considered, by inserting unsteady 


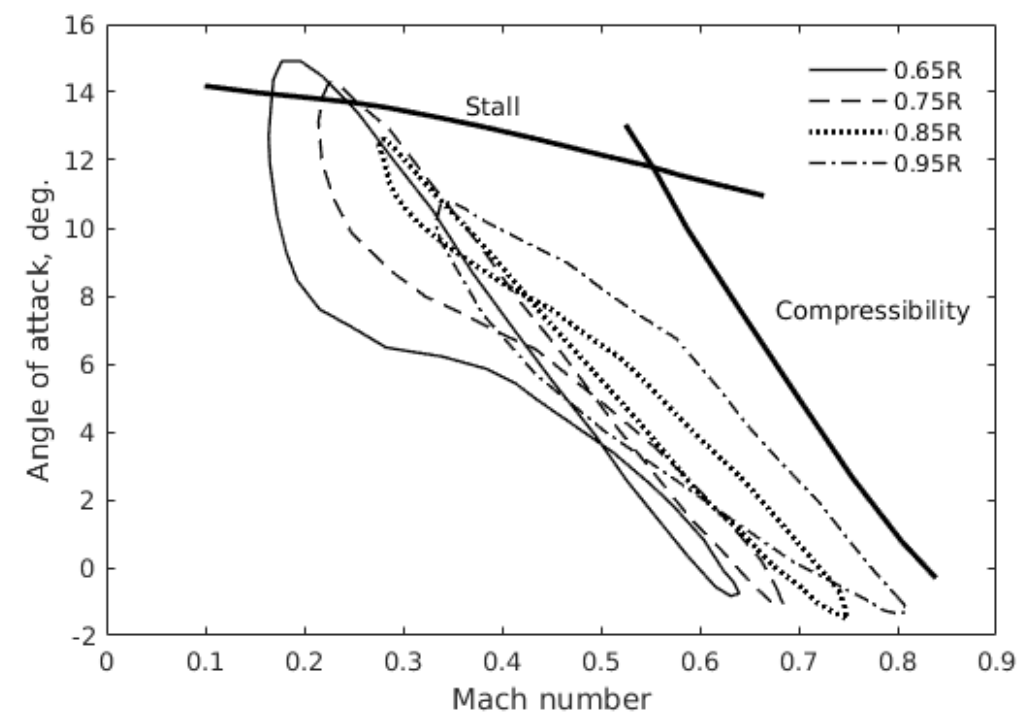

Figure 1: Operating conditions for blade sections (data from Ref. [1, p.296]).

models in the optimization loop and directly evaluating the cycle of lift, drag and moment coefficients for each design, as done in [15]. However, this approach is very expensive from a computational point of view, and the accurate prediction of time-varying aerodynamic loads of pitching and plunging airfoil is still a challenge. In most cases, one can resort to a multipoint approach, where the evaluation of the aerodynamic performance is obtained by means of steady models computed at different azimuth angles $\psi$, see Fig. 2. This is the approach followed in several works on optimization of airfoils for rotorcraft, see Refs. [16-18].

This approach could be used to account for the variability of $\alpha-M$ along the azimuth, but the $\alpha-M$ set changes as the aircraft flies at a different advancing ratio or at a different disk loading. However, the increase of the number of points that compose the objective function may be difficult to manage by the optimization task [18], causing problems in the numerical procedures and in the methods to analyse the results. An help in this sense 


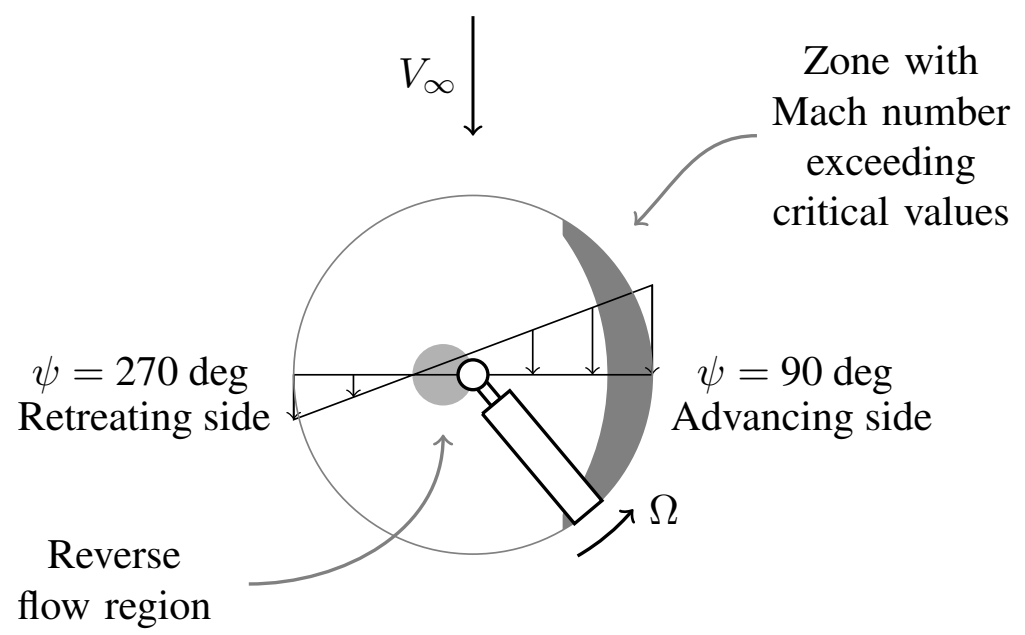

Figure 2: Scheme of incident speed on the rotor blade in forward flight.

could come by the employment of the robust optimization approach presented in Ref. [19], that allows to look for an airfoil that is minimally sensitive to the variation of operating conditions. This approach is the one pursued here. However, the robust optimization represents a change of paradigm with respect to the deterministic one, because instead of optimizing a single figure of merit or a collection of figures of merit, it optimizes a statistical measure of the figure of merit or a collection of statistical measures, taking into account the fact that the airfoil will operate in a variable uncertain environment. This change requires a significant change in the problem formulation.

This work extends the application of robust optimization methods based on consideration of uncertainties for the optimization of helicopter rotor airfoils, initially presented in Ref. [19] for hover, to the more complex and challenging case of forward flight. In the following sections, the optimization problem of improving the aerodynamic performance of fixed airfoils is tackled from both deterministic and robust points of view. The robust approach 


\begin{tabular}{lccccccc}
\hline Position & Case \# & $\alpha[\mathrm{deg}]$ & $M[-]$ & $R e[-]$ & $C_{L}[-]$ & $C_{D}[$ counts $]$ & $C_{M}[-]$ \\
\hline Advancing & 1 & -1.5 & 0.75 & $4.6 \mathrm{e} 6$ & -0.025 & 86.97 & 0.02 \\
Retreating & 2 & 12.5 & 0.28 & $1.7 \mathrm{e} 6$ & 1.411 & 191.3 & 0.03 \\
\hline
\end{tabular}

Table 1: Aerodynamic coefficients of Bo-105 NACA 23012 airfoil at radial station $r / R=$ 0.85 on the advancing and retreating sides.

by taking into account the uncertainty of the operating conditions has the potential to lead to airfoils that are more suitable for rotorcraft.

In particular, a two-point optimization problem is set up, considering the two most representative conditions of forward flight: the retreating and advancing blade conditions (see Fig. 2). The idea of using two conditions is aligned with the choice made by other authors dealing with the forward flight optimization, see $[16,18]$, and allows a thorough comparisons of the two optimization approaches presented here. However, the employment of a larger number of blade sections would be conceptually simple, adding only a computational burden to what is presented here.

The optimization will investigate the blade section of the Bo105 rotor [20, 21] used in the full-scale wind tunnel experiments at NASA Ames. The airfoil of the blades used in the test was the NACA 23012. Here the airfoil at the radial station $r / R=0.85$ is considered and the operating conditions for advancing and retreating sides are taken from the corresponding "figure-ofeight" in the $(M, \alpha)$-plane from Ref. [1] presented in Fig. 1. Both operating Mach numbers and the corresponding constraints are reported in Tab. 4.

The evaluation of the performance of the optimal shapes over the azimuth angle is performed in the post-processing phase with steady models. To motivate the choice of steady models, section 2 discusses the comparison between unsteady CFD predictions and steady computations. 
In the deterministic case presented in section 3 , the objective of the optimization is to minimize the drag, while maintaining a level of lift that guarantees satisfaction of trimming condition. In the case of robust design, a single condition is not considered, and the trimming condition cannot be applied to a range of values for the angle of attack. Thus, the idea is to optimize the lift-to-drag ratio as a measure of the performance of the airfoil, impose an inequality constraint on the lift coefficient to guarantee a sufficient level of lift, and then check after optimization that the trimming condition can be satisfied. This approach is used in section 4, and results coming from deterministic and robust optimization are presented and compared in section 5. Because in both cases only two points over the azimuth angle are considered in the optimization loop, an a posteriori assessment of the performance over the azimuth angle is presented in section 6. Finally, conclusions and perspectives are drawn in section 7 .

\section{Unsteady vs steady CFD simulations for the evaluation of loads in forward flight}

This section investigates how correct is the idea of using steady simulations of blade airfoils at different azimuth angles for the optimization in forward flight, considering that the real flow condition is fully unsteady. The investigation begins with the simulation of the unsteady loads acting on the reference airfoils, i.e. the NACA 23012 and the NACA 0012 airfoils, during a combined pitching motion and periodic variation of Mach number, representative of the conditions met by rotor airfoils during forward flight.

In the literature, only few works have considered a translation along the freestream direction which is necessary to simulate the speed fluctuation encountered by the blade section [22]. Among these, Refs. [23, 24] have been 
devoted to the experimental investigation of the effects of coupled oscillations in angle of attack and freestream speed, as it occurs in the real flow around the rotor blade. These studies have tackled conditions below static stall, and through stall as well. A few theoretical aerodynamic models based on the extension of Theodorsen's method [14, p.431] are presented in Ref. [25] for an airfoil undergoing a combination of harmonic pitching, plunging, and fore-aft motion in incompressible flow.

\subsection{Unsteady CFD set-up and validation}

The simulation is performed by means of the $\mathrm{SU}^{2}$ software suite [30]. In particular, the unsteady Reynolds-Averaged Navier-Stokes (RANS) equations together with the Spalart-Allmaras model [31] are used to simulate the flowfield around the airfoil. The numerical grid is a C-type, structured mesh (Fig. 3) with 77792 rectangular cells. The number of airfoil wrap-around points is 128 , while the normal points are 256 . The grid extends \pm 30 chords around a unit chord airfoil (Fig. 3b) and the the wall $y^{+}$is equal to one, since a wall log-law is not used in the simulation. Grid convergence studies are reported in [32].

The time-varying freestream velocity and angle of attack have the following time dependency

$$
\begin{aligned}
& V(t)=V_{\infty}(1+\lambda \sin (\omega t)) \\
& \alpha(t)=\alpha_{0}+\Delta \alpha \sin (\omega t+\bar{\psi})
\end{aligned}
$$

where $V_{\infty}$ is the mean freestream speed, $\lambda$ is the percentage of variation in the freestream speed, $\omega$ is the angular velocity of the fluctuations, $\alpha_{0}$ is the mean angle of attack, $\Delta \alpha$ is the amplitude of the angle of attack oscillation, and $\bar{\psi}$ is the phase angle. The freestream velocity direction is not modified during pitch oscillations. 


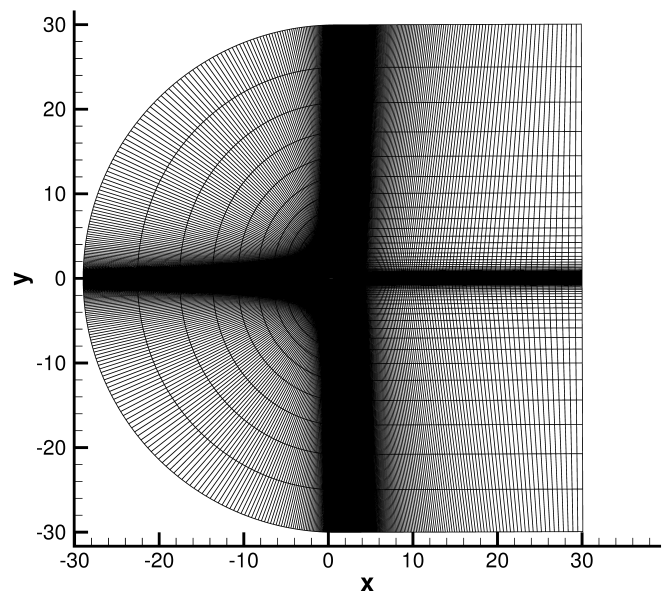

(a) C-mesh.

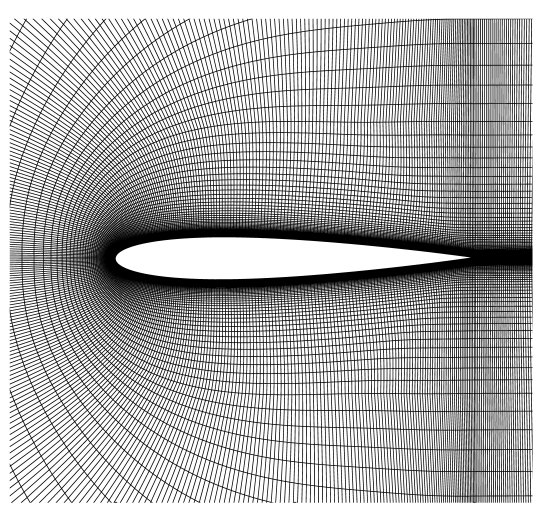

(b) Close-up on the airfoil.

Figure 3: Mesh for unsteady CFD computations.

To obtain the sinusoidal variation to the freestream velocity of Eq. (1), a time-varying translation of the airfoil along the direction of the mean angle of attack $h(t)$ is performed with the following time dependency

$$
h(t)=-\frac{V_{\infty} \lambda}{\omega} \cos (\omega t) .
$$

The time-varying translation and pitching are implemented with a volumetric rigid displacement applied to each cell of the mesh in each iteration of the physical time. To be consistent with the experimental data, it is necessary to match the reduced frequency $k=\omega c / 2 V_{\infty}$, where $c$ is the airfoil chord.

The CFD setting is validated against experimental and computational measurements. Different test cases are considered for the validation. Here, the focus is on the experimental data taken from Ref. [23] for the case of the NACA 0012 airfoil undergoing the speed fluctuation in Eq. (1). The corresponding operating conditions are reported in Tab. 2. The computations have been performed considering 100 intervals for each period $T=2 \pi / \omega$, and 800 steps in the dual-time iteration. The number of steps in dual time 


\begin{tabular}{cccccccc}
\hline$\alpha_{0}[\mathrm{deg}]$ & $\Delta \alpha[\mathrm{deg}]$ & $V_{\infty}[\mathrm{m} / \mathrm{s}]$ & $R e$ & $M_{\infty}$ & $\lambda$ & $k$ & $\bar{\psi}[\mathrm{deg}]$ \\
\hline 6. & 0. & 25 & $4 \cdot 10^{5}$ & 0.07 & 0.114 & 0.101 & 0 \\
\hline
\end{tabular}

Table 2: Operating conditions for validation of SU2 model with experimental data from Ref. [23].

are set to 800 to guarantee that the residuals of the equations are always lower than 1e-4 for every time step. It is noted that the simulation with time-varying freestream Mach number requires a streamwise translation of the airfoil with amplitudes depending on the chosen variation of speed, but also on the reduced frequency. In some cases, the amplitude of this motion may exceed 5 chords, therefore it has been found necessary to both reduce the time steps — to apply a small translation at each outer iteration and increase the number of dual time steps - to guarantee time-accuracy. Figure 4 presents the lift ratio $L / L_{s t}$ between the unsteady lift $L$ and the steady value $L_{s t}$ plotted against the azimuth angle $\psi=\omega t$. A very good agreement is found between the CFD result obtained with the $\mathrm{SU}^{2}$ software and the experimental data, with a maximum error affecting the amplitude of the lift ratio of $6 \%$.

Additionally, the numerical data presented in Ref. [27] for the NACA 0006 airfoil have been compared to the results obtained with the unsteady CFD set-up presented in the preceding section. In this case, it was possible to compare also the pitching moment. The resulting aerodynamic coefficients are presented in Fig. 5. More extensive details on the validation can be found in Ref. [32] where results of combined pitch and freestream velocity oscillations can be found. As a final remark, it is noted that experimental data on this kind of oscillating airfoil are scarce and that measurements of the unsteady drag coefficients are not assessed in the respective literature. 


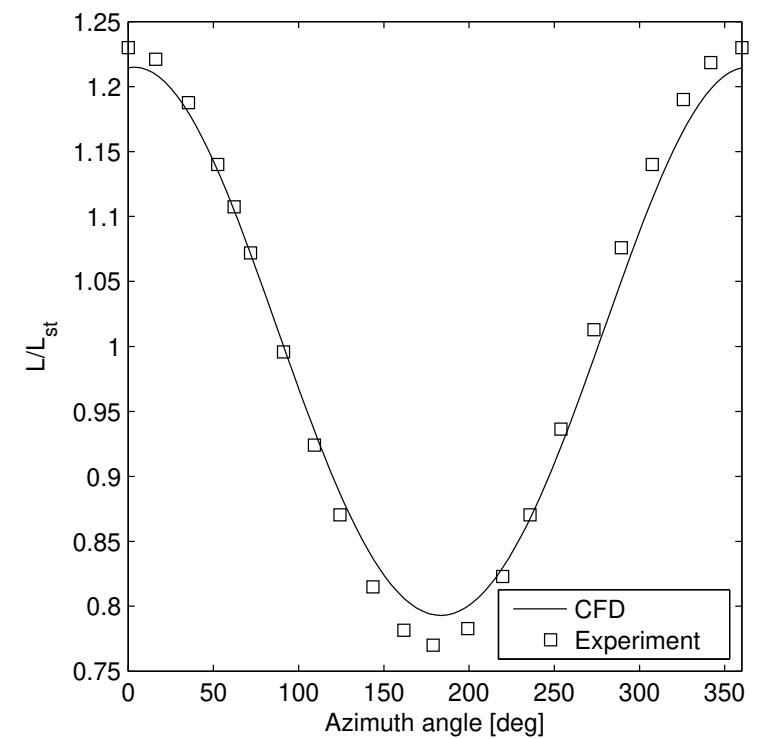

Figure 4: Lift ratio $L / L_{s t}$ as a function of the azimuth angle $\psi=\omega t$ for an airfoil undergoing freestream Mach number variation (Eq. (1)). Maximum error if CFD SU2 model equal to $6 \%$.

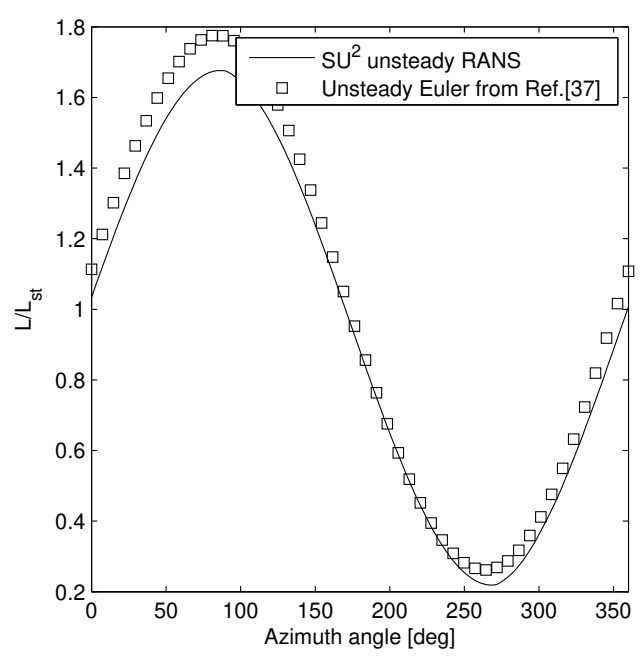

(a) Lift coefficient.

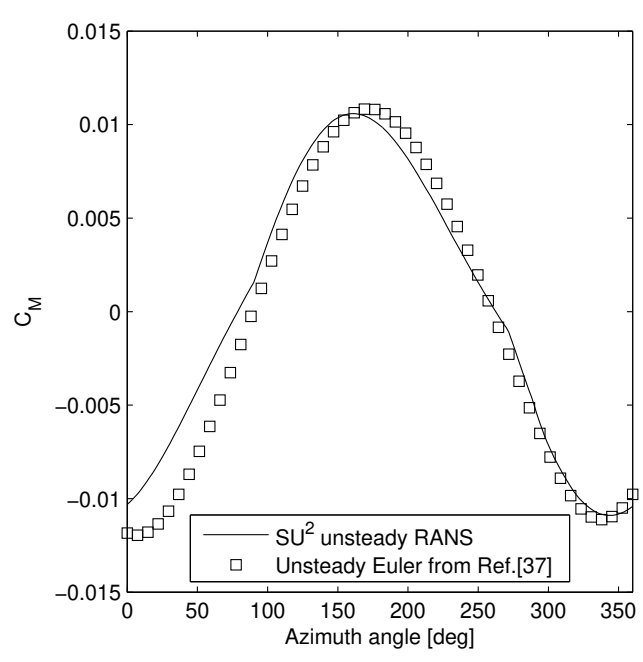

(b) Moment coefficient.

Figure 5: Lift and moment coefficient for $\alpha=2+2 \sin (\omega t) \operatorname{deg}, k=0.2$.

\subsection{Comparison of steady and unsteady simulation}

The estimate of the lift and drag coefficients obtained with the CFD set-up are compared to the steady approximation used in the optimization 
problem. This comparison is obtained by computing the aerodynamic coefficients by means of an unsteady RANS simulation presented in the preceding section and different steady models:

- for the advancing side, an Euler equations simulation with $\mathrm{SU}^{2}$ is performed, with the set-up that will be later presented for the optimization problem (section 3.2). Because the Euler equations are used, a correction associated with viscous effects is added to the inviscid $\mathrm{SU}^{2}$ estimate combining the van Driest II method and a form-factor correction as presented in Ref. [34];

- for the retreating side, the XFOIL solver is adopted

- for the remaining intermediate points, the MSES code is used.

Steady state solutions were computed at eight equidistant azimuth stations starting from azimuth 0 (see Figure 2) for the airfoil at $r / R=0.85$. The

\begin{tabular}{lcccccc}
\hline & \multicolumn{3}{c}{ NACA 0012 } & \multicolumn{3}{c}{ NACA 23012 } \\
& Unsteady & Steady & Error & Unsteady & Steady & Error \\
\hline $\operatorname{avg}\left(C_{L}\right)$ & 0.591 & 0.588 & $0.5 \%$ & 0.756 & 0.769 & $1.8 \%$ \\
$\operatorname{avg}\left(C_{D}\right)[$ counts] & 126.61 & 110.18 & $12 \%$ & 121.26 & 101.51 & $16 \%$ \\
\hline
\end{tabular}

Table 3: Average drag coefficient and lift coefficient obtained from steady and unsteady computations for the NACA 23012 airfoil.

results for the NACA 0012 airfoil and NACA 23012 airfoil are presented in Fig. 6 and Fig. 7, respectively. The average aerodynamic coefficients over the azimuth angle are presented in Tab. 3. An excellent agreement is observed with regard to the lift coefficient, both in terms of average value and amplitude oscillations. The difference between the unsteady CFD-based 
average and the steady value is less than 1\% for the NACA 0012 airfoil and less than $2 \%$ for the NACA 23012 airfoil. In terms of drag coefficient, the discrepancy between the unsteady results and the steady approximation is more pronounced. The unsteady wake effects are completely neglected in the steady approximation and they greatly affect the drag coefficient, more so than in the case of the lift coefficient. This is most likely the reason for this reduction in accuracy. Nevertheless, the average values of the drag coefficient differ by $12 \%$ and $16 \%$ for the NACA 0012 and NACA 23012, respectively. The magnitude of the error for the steady approximation is encouraging, and the different steady models are considered an acceptable solution to significantly reduce the computational cost of the optimization loop, both deterministic and robust. Additionally, Figs. 6-7 show that the two azimuth angles selected are reasonably representative of the range of drag coefficients met by the airfoil during each revolution. The average drag coefficient per rotor turn that influences the torque can be estimated using the steady state approximation at a fraction of cost necessary to run a fully unsteady analysis.

The usage of this variable fidelity approach allows for an efficient usage of computational resources, as pointed out by Ref. [35]. This is especially important for robust optimization that are always computationally intensive [19]. In addition, the work presented in Ref. [15] backs up this conclusion. In fact, Reference [15] demonstrates that optimal solutions are not greatly affected by the use of either an unsteady or steady model, as long as the reduced frequency is moderate. This is often the case for rotorcraft where $1 /$ rev frequency is typically below a reduced frequency of 0.06 [36, p. 449]. For this specific Bo-105 rotor the 1/rev reduced frequency, related to freestream velocity variation, is equal to 0.034 , and the highest reduced 


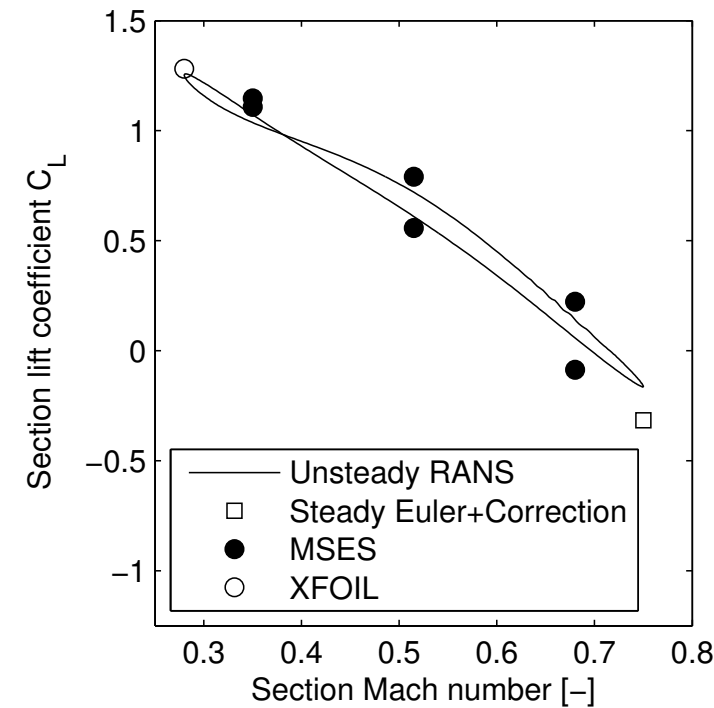

(a) Lift coefficient.

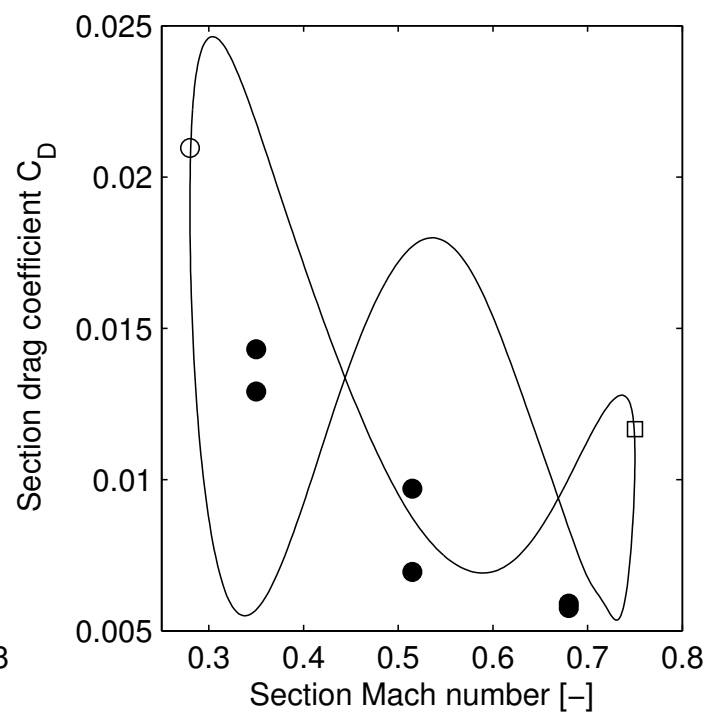

(b) Drag coefficient.

Figure 6: Cycle of lift and drag coefficient of the NACA 0012 airfoil.

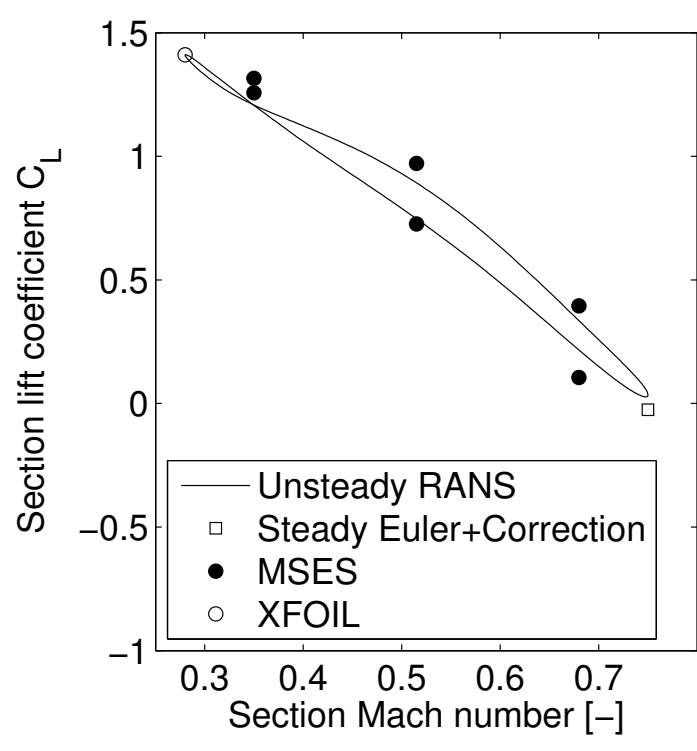

(a) Lift coefficient.

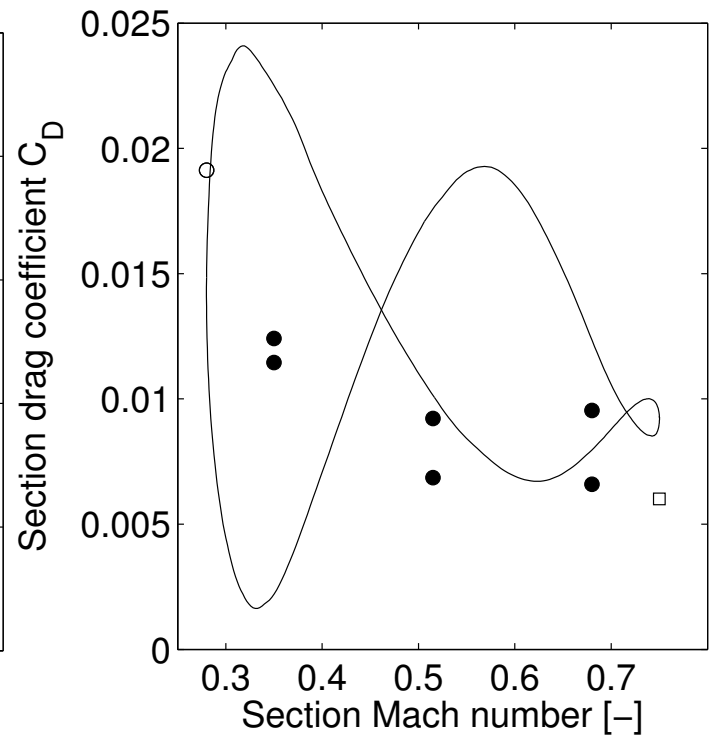

(b) Drag coefficient.

Figure 7: Cycle of lift and drag coefficient of the NACA 23012 airfoil. 
frequency reached by the angle of attack is 0.12 (see section6).

\section{Deterministic forward flight optimization}

The deterministic optimization of a helicopter rotor airfoil in forward flight consists typically in the minimization of the drag coefficient $C_{D}$ ensuring the satisfaction of constraints on the lift $C_{L}$ and moment $C_{M}$ coefficients. Examples of such an approach are given in Refs. [16-18]. The constraint on lift coefficient is set to maintain the trim condition of the entire helicopter and reduce the interaction with blade dynamics.

Here, a target lift coefficient $\overline{C_{L}}$ is set to consider a specific trim condition, and the moment coefficient is bounded with a given threshold $\overline{C_{M}}$ to avoid excessive loading on the blade pitch link. The objectives on the advancing and retreating sides are conflicting with one another. In fact, an optimal shape for the advancing side would be a mildly cambered airfoil to postpone drag rise to higher Mach number values, whereas the adoption of a highlycambered airfoil on the retreating side could better adapt to higher angle of attack, for instance by means of a nose-droop.

In mathematical terms, two single-objective optimization problems may be formulated as follows

$$
\begin{aligned}
\text { minimize: } & C_{D_{i}}(\boldsymbol{x}) \\
\text { subject to: } & C_{L_{i}}(\boldsymbol{x})=\overline{C_{L_{i}}} \quad\left|C_{M_{i}}(\boldsymbol{x})\right| \leq \overline{C_{M_{i}}}
\end{aligned}
$$$$
\text { by changing: } \quad \boldsymbol{x}
$$

where $\boldsymbol{x}$ are the design variables, and the subscript is related to the advancing or retreating position as shown in Table 5. .

The values of the lift constraint $\overline{C_{L_{i}}}$ are set for each side with the intent of producing a design that improves the performance of the baseline 
NACA 23012 airfoil. The aerodynamic coefficients of the NACA 23012 airfoil in the operating conditions are presented in Tab. 1. It is possible to note that the NACA 23012 airfoil provides a slightly negative lift on the advancing side. At high advance ratio it is possible that the outer portion of a helicopter blade can operate at slight negative lift. However, to avoid any possible confusion it has been chosen to change $\overline{C_{L_{1}}}$ into a slightly positive value set to generate lift. Furthermore, the constraint on the moment coefficient has been enlarged with respect to the value of the NACA 23012 airfoil to give more freedom to the design optimization and explore design solutions which may have a larger moment coefficient but better performance in the transonic condition.

To complete the scenario of deterministic optimization problem, a oneobjective optimization with a trade-off performance is presented. The optimization problem in this case is defined as follows

$$
\begin{aligned}
\text { minimize: } & \frac{1}{2} \sum_{i=1}^{2} w_{i} C_{D_{i}}(\boldsymbol{x}) \\
\text { subject to: } & C_{L_{i}}(\boldsymbol{x})=\overline{C_{L_{i}}} \quad\left|C_{M_{i}}(\boldsymbol{x})\right| \leq \overline{C_{M_{i}}} \quad i=1,2
\end{aligned}
$$

by changing: $\boldsymbol{x}$,

where $w_{i}$ are weights chosen from the drag coefficient of the optimal airfoils obtained in the single-point optimization cases (see Tab. 4). The result of this optimization is a compromise solution between advancing and retreating sides that is used as a reference trade-off optimal solutions.

\subsection{Design variables}

Particular attention is drawn to the design variables. To describe an airfoil shape with a finite set of variables, the Class/Shape function Transformation (CST) [37] is used. The parameterization is well-defined by spec- 


\begin{tabular}{lccccc}
\hline Case & $M[-]$ & $R e[-]$ & $\overline{C_{L}}$ & $\overline{C_{M}}$ & $w_{i}[1 /$ counts $]$ \\
\hline 1. & 0.75 & $4.6 \mathrm{e} 6$ & 0.025 & 0.08 & $1 / 2$ \\
2. & 0.28 & $1.7 \mathrm{e} 6$ & 1.411 & 0.04 & $1 / 160$ \\
\hline
\end{tabular}

Table 4: Operating conditions and aerodynamic constraints.

ifying two functions: a geometry class function $C$ and a shape function $S$ that defines the particular shape of the geometry. In this case, the airfoil shape is decomposed into the camber mean line $\zeta_{c}$ and the normal thickness $\zeta_{t}$ distributions. The CST is applied to $\zeta_{c}$ and to $\zeta_{t}$ of the airfoil and it reads

$$
\begin{aligned}
& \zeta_{c}(\chi)=C(\chi) S(\chi)+\chi \zeta_{T E_{c}}=C(\chi) \cdot \sum_{i=0}^{n} A_{i}^{c} S_{N, i}(\chi)+\chi \zeta_{T E_{c}} \\
& \zeta_{t}(\chi)=C(\chi) S(\chi)+\chi \zeta_{T E_{t}}=C(\chi) \cdot \sum_{i=0}^{n} A_{i}^{t} S_{N, i}(\chi)
\end{aligned}
$$

where $\chi=x / c$ is the non-dimensional chordwise coordinate, the coefficients $A_{i}^{c}, A_{i}^{t}$ define the Bernstein polynomial coefficients of the camber line and thickness distributions respectively, $S_{n, i}$ is the $i$-th term of the Bernstein polynomial of order $N$ [37].

The thickness distribution is taken in perpendicular direction with respect to the camber mean line. Then, the camber line and thickness distribution are such that the upper surface $\zeta_{u}$ and lower surface $\zeta_{l}$ are obtained applying the thickness perpendicular to the camber line, as follows

$$
\begin{aligned}
\zeta_{u} & =\zeta_{c}+\zeta_{t} \cos (\epsilon) \\
\zeta_{l} & =\zeta_{c}-\zeta_{t} \cos (\epsilon),
\end{aligned}
$$

where $\epsilon=\arctan \left(\frac{\mathrm{d} \zeta_{c}}{\mathrm{~d}_{\chi}}\right)$. Please note that also the $\chi$ coordinate of the resulting airfoil will be affected by this summation of vectors. 
The coordinate of the camber mean line at the trailing edge is set to zero to consider a closed trailing edge and null geometric angle of attack. In addition, the class functions for the camber line $C_{c}$ and the thickness distribution $C_{t}$ are defined as follows

$$
C_{c}(\chi)=\chi(1-\chi) \quad C_{t}(\chi)=\sqrt{\chi}(1-\chi)
$$

While the class function for the thickness distribution is the one suggested in Ref. [37] to define a rounded nose distribution close to the leading edge, the class function for the camber presents a linear term to avoid vertical slope of the camber distribution at the leading edge. In addition, the linear term of the class function permits to directly relate the first coefficient of the shape function $A_{0}^{c}$ to the slope of the camber line at the leading edge.

The coefficients of theshape function $S(\chi)$ are the design variables $\boldsymbol{x}_{s}$ of the optimization problem. A convergence study, suggested the employment of 4 design variables for each distribution that yields a total of 10 design variables.

The order of the polynomial could be chosen based on a convergence analysis of the CST. To do this, an extensive numerical campaign has been performed for representing a group of helicopter rotor airfoils - e.g. NACA23012, SC1095, SC1094, HH02 — by using an increasing polynomial order (for further details see Ref. [19]).

In addition to the coefficients describing the shape of the airfoil, the angle of attack can be regarded as a design variable. In fact, it is possible to solve the constrained optimization problem stated in Eq. (4) by acting on the airfoil design variables $\boldsymbol{x}_{s}$ to minimize the drag coefficient and on the angle of attack to track the target lift coefficient inside two nested loops. The underlying idea is an analogy with the blade collective pitch control: to increase thrust it is necessary to act on the collective pitch, which changes 
the angle of attack of the blade section.

Thus, each airfoil tested in the optimization loop is obtained with a specific set $\boldsymbol{x}_{s}$, and its performance is evaluated computing the angle of attack $\alpha$ that provides the desired lift coefficient. The nested optimization loops used to implement this procedure are described in detail in Section 3.3. Thus, the resulting set of design variables is $\boldsymbol{x}=\left\{\boldsymbol{x}_{s}, \alpha\right\}$.

\subsection{Aerodynamic models}

A model describing the aerodynamic loads acting on the airfoil is necessary to compute the objective function of the optimization problem. The aerodynamic models used in this optimization problem are different for each side of the blade in order to capture the specific features of the flow in such different operating conditions, while limiting the computational effort.

On the retreating side featuring subsonic condition below the static stall boundary, XFOIL is adopted, which is an aerodynamic code which couples panel and integral boundary layer methods for the analysis of subsonic, isolated airfoils [38]. This code is chosen because it provides a fast and sufficiently accurate estimation of the aerodynamic force coefficients for a two-dimensional section in the range of angle of attack considered in this problem. Please note that the highest value of the angles of attack in the range is below the static stall limit for most airfoils. Here, the transition of the boundary layer is predicted by applying the $\mathrm{e}^{n}$ criterion [39], while compressible effects for low Mach numbers are taken into account by the Karman-Tsien correction. For the adjustable transition parameter $N_{\text {crit }}$ the value of 9 gives satisfactory results for helicopter flows, as shown in Ref. [40].

For fully developed transonic conditions, such as the one experienced in forward flight, a solver based on Euler equation, able to compute the drag rise caused by the appearance of shock wave was considered sufficient to reach 
the optimization objective of a shock-free or minimum shock airfoil. So, the inviscid solver of the $\mathrm{SU}^{2}$ suite can be used. In this case no boundary layer is modelled since the viscous drag is not considered as part of the objective of the optimization on the advancing side.

For the computation inside the optimization loop, a two-dimensional coarse structured mesh of 20480 rectangular cells is used (see Fig. 8). The mesh extends \pm 25 chords around the airfoil. For each airfoil in the optimization loop, the computation runs for 500 iterations starting from a baseline solution of the NACA0012 airfoil. The mesh is deformed by displacing the airfoil boundary cells according to the new geometry and by translating the displacement to the volume cells with a method based on the finite element method discretization of the linear elasticity equations [30]. The element stiffness is set inversely proportional to the wall distance, and 500 smoothing iterations are applied to get a regularly deformed mesh.

The computational cost for each steady evaluation of the figure of merit using the high fidelity Euler model is about fifteen times that of running a solution with the low fidelity XFOIL solver.

\subsection{Optimization algorithm}

To solve the optimization problem in Eq. (4), a Non-dominated Sorting Genetic Algorithm [43] is chosen for its ability to explore the design space. Evolutionary algorithms have been employed to tackle many engineering optimization problems. Applications to aerodynamic optimization problems can be found for instance in Refs. [44-46]. Main advantages of such approaches are the possibility to tackle multi-objective problems without scalarization and to exhaustively explore the design space. On the other hand, they are very expensive from a computational point of view because they require many computations of the objective function, even in region of 


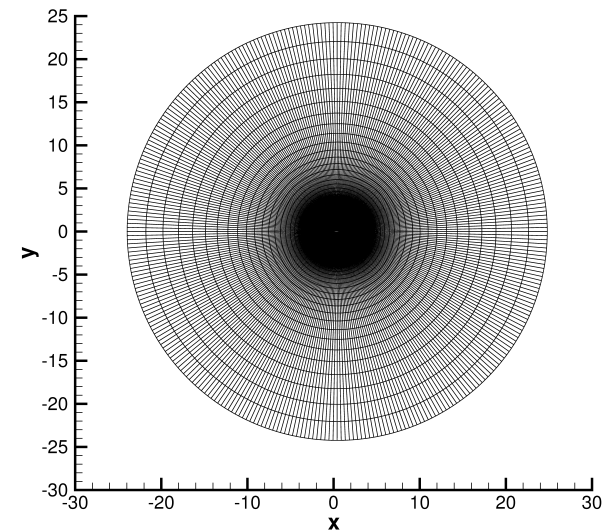

(a) O-mesh.

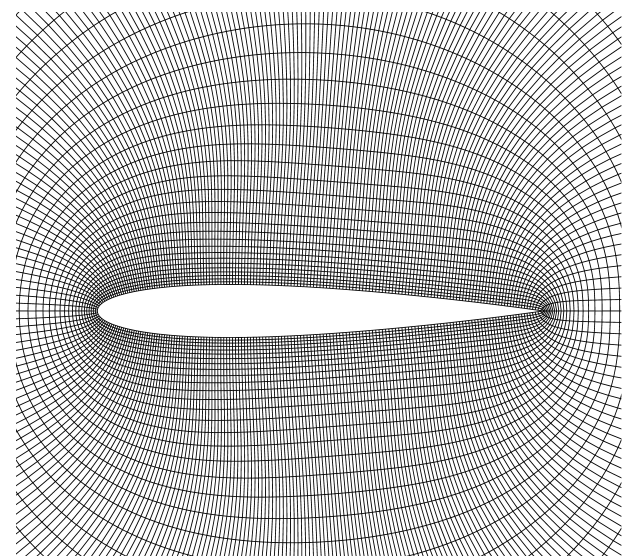

(b) Zoom.

Figure 8: Coarse structured grid (20480 cells) for $\mathrm{SU}^{2}$ Euler inviscid computations inside the optimization loop.

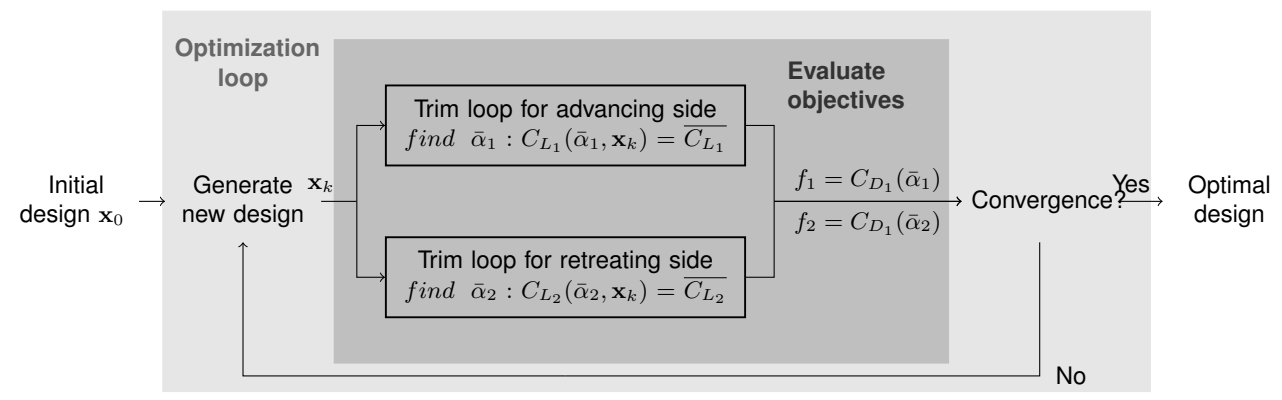

Figure 9: Outer NSGA loop for $\boldsymbol{x}_{s}$ and inner trim loop to determine $\bar{\alpha}$.

the domain where designs with poorer performance are present.

Inside the genetic algorithm loop, an inner loop is nested to deal with the lift coefficient constraint (see Fig. 9). In particular, for each design vector $\boldsymbol{x}_{s, k}$ of the $k$-th iteration in the genetic loop, a secant method is used to find the angle of attack $\bar{\alpha}$ that guarantees $C_{L, k}=\overline{C_{L}}$. The $i$-th iteration of the secant loop reads

$$
\alpha_{i}=\alpha_{l_{i}}+\frac{\alpha_{r_{i}}-\alpha_{l_{i}}}{C_{L}\left(\alpha_{b_{i}}\right)-C_{L}\left(\alpha_{a_{i}}\right)}\left(\overline{C_{L}}-C_{L}\left(\alpha_{a_{i}}\right)\right)
$$

where $\alpha_{l_{i}}$ and $\alpha_{r_{i}}$ are the left and right boundaries of the interval in which 
the angle of attack is sought and they satisfy: $C_{L}\left(\alpha_{r_{i}}\right)>\overline{C_{L}}$ and $C_{L}\left(\alpha_{l_{i}}\right)<$ $\overline{C_{L}}$. The left and right values are updated according to the value of the lift coefficient for the current $\alpha_{i}$ : if $C_{L}\left(\alpha_{i}\right)>\overline{C_{L}}$, then $\alpha_{r_{i+1}}=\alpha_{i}$; if $C_{L}\left(\alpha_{i}\right)<\overline{C_{L}}$, $\alpha_{l_{i+1}}=\alpha_{i}$, and if $C_{L}\left(\alpha_{i}\right)=\overline{C_{L}}$, the loop breaks.

The method converges in a few iterations, owing to the quasi-linearity of lift coefficient for most airfoils in the vicinity of considered values of the angle of attack. Lack of convergence within a few iterations is not critical, because this happens for airfoils with poor values of the lift coefficient that should be discarded anyway. The result of the two nested loops is a set of optimal solutions defined by a design vector $\left\{\boldsymbol{x}_{s}, \alpha\right\}$. It is noted that the inner trim loop targets the equality constraint on the lift coefficient, but the resulting design vector may not satisfy the moment coefficient constraint.

Additionally, a constraint on the minimum allowable airfoil thickness as been considered to obtain airfoils that satisfy also structural requirements. The lower limit for thickness was set at $10 \%$ given that airfoils used at this radial station can have a thickness as low as 9.2\%. Airfoils with a thickness below the threshold were simply discarded during the creation of a new generation of individuals.

\section{Robust optimization problem}

The objective of robust optimization is to design an airfoil that is minimally sensitive to the variation of the operating conditions. In this case, it is considered that the operating conditions at which the blade section will operate are affected by the uncertainty arising due to modelling assumptions of physical parameters necessary at the design stage.

Due to the uncertainty, the objective function $f$ is no longer only a function of the design variables $\boldsymbol{x}$, but it also depends on the uncertain variables 
$\boldsymbol{\xi}$. Within this uncertain framework a minimization with a target lift coefficient at a specific design condition loses its meaning. However, a robustly optimal airfoil with a satisfactory lift-to-drag ratio in a range of conditions could be used to trim the aircraft at a specific lift coefficient, keeping always a low drag coefficient. In addition, if the robustness of the lift-to-drag ratio will translate into the drag coefficient at a specific target lift coefficient, a robust shape would ensure less variability of the required power due to aerodynamic drag.

In particular, for the problem under analysis, the following objectives are defined for each side of the blade.

1. On the advancing side, the rotor blade encounters transonic flow, so airfoils should typically be designed to delay drag divergence to higher Mach numbers $[13,14]$. Thus, the lift-to-drag ratio $C_{L} / C_{D}$ is the objective $f_{1}$ of the optimization: $f_{1}=C_{L} / C_{D}$, with the constraint of providing a lift coefficient equal to or greater than the value of the baseline airfoil.

2. On the retreating side, the increase of the lift coefficient is typically sought [13]: for the blade at $180 \mathrm{deg}$ the ratio $C_{L}^{3 / 2} / C_{D}$ is maximized, which is a measure related to the rotor figure of merit [14] and privileges the lift coefficient over the drag coefficient, that is $f_{2}=C_{L}^{3 / 2} / C_{D}$.

Finally, the constraints on the moment coefficient and minimum thickness used in the deterministic case are still considered.

In mathematical terms, the resulting optimization problem can be stated 
as:

$$
\begin{array}{rc}
\text { maximize : } & \mu\left(f_{i}\left(\boldsymbol{x}_{s}, \boldsymbol{\xi}\right)\right) \\
\text { and minimize : } & \sigma^{2}\left(f_{i}\left(\boldsymbol{x}_{s}, \boldsymbol{\xi}\right)\right) \\
\text { subject to : } & \mathbf{g}\left(\boldsymbol{x}_{s}, \boldsymbol{\xi}\right) \leq \mathbf{0} \\
\text { by changing : } & \boldsymbol{x}_{s},
\end{array}
$$

where the constraints are collected in vector $\mathbf{g}$. The objective functions of Eq. (10) are related to the statistics of the figure of merit $f_{i}$ : the mean value $\mu$ and the variance $\sigma^{2}$. The design variables in this case are only $\boldsymbol{x}_{s}$, while the angle of attack is considered as one of the uncertain parameters in the robust design problem.

Indeed, the angle of attack $\alpha$ and the Mach number $M$ encountered by the two-dimensional section of the blade are considered as uncertain, because they are affected by uncertainty on the modelling of the physical parameters considered in the design stage. Both aerodynamic and structural uncertainties, may affect the value of the angle of attack and Mach number. Following a probabilistic framework, the uncertain variables are modelled as uniformly distributed random variables around a nominal value. The nominal conditions are reported in Tab. 1 and the uncertainty band is set to $5 \%$ for the Mach number and for the angle of attack.

The statistics of the performance $f_{i}$ are computed by means of an uncertainty propagation technique, a method to propagate the uncertainty affecting the operating conditions into the quantity of interest $f$.

\subsection{Uncertainty quantification}

A probabilistic framework is employed to deal with the uncertain input data. In this context, the stochastic input quantities $\boldsymbol{\xi}$ are treated as independent continuous random variables. The random vector $\boldsymbol{\xi}$, whose dimension 
is equal to the number of uncertain variables $n_{\xi}$, belongs to the probability space $(\Omega, \mathcal{F}, P)$, composed of the sample space $\Omega$, the $\sigma$-algebra $\mathcal{F}$ of the subsets of the events, and a probability measure $P$. It essentially maps the samples in $\Omega=[0,1]^{n_{\xi}}$ into the random outcomes $\boldsymbol{\xi} \in \Xi$, and it is characterized by the probability density function $p_{\xi}(\boldsymbol{\xi})$. The output of the system is then a stochastic variable, and therefore the performance $E(\mathbf{y}, \boldsymbol{\xi})$, which is a function of the solution, is a stochastic variable as well. In the robust optimization, procedure the interest is in reconstructing the mean value and the variance of the quantity of interest; in the following, the stochastic output variable is referred to as $u(\mathbf{y}, \boldsymbol{\xi})$, while its deterministic realization is $\tilde{u}_{i}\left(\mathbf{y}, \boldsymbol{\xi}_{i}\right)$. Let us define the expected value of the stochastic variable $u$ as follows

$$
\langle u\rangle=\int_{\Xi} u(\mathbf{y}, \boldsymbol{\xi}) p_{\xi}(\boldsymbol{\xi}) \mathrm{d} \xi,
$$

with $p_{\xi}(\boldsymbol{\xi})=\prod_{i}^{n_{\xi}} p_{\xi_{i}}\left(\xi_{i}\right)$ being the joint probability of the independent input variables. Let us define the inner product operator of two stochastic variables $u$ and $v$ with respect to the joint probability (i.e. the covariance for independent $u$ and $v$ )

$$
\langle u, v\rangle=\int_{\Xi} u(\mathbf{y}, \boldsymbol{\xi}) v(\mathbf{y}, \boldsymbol{\xi}) p_{\xi}(\boldsymbol{\xi}) \mathrm{d} \xi
$$

The mean and variance then read

$$
\begin{aligned}
\mu(u) & =\langle u\rangle \\
\sigma^{2}(u) & =\langle u, u\rangle-\langle u\rangle^{2}
\end{aligned}
$$

To reconstruct the quantities in Eq. (11), a Polynomial Chaos (PC) expansion method [47] is used. In fact, under specific conditions, a stochastic process can be expressed as a spectral expansion based on suitable orthogonal polynomials, with weights associated to a particular probability density 
function. The first study in this field is the Wiener process that was later developed in Ref. [47]. The basic idea is to project the variables of the problem onto a stochastic space spanned by a complete set of orthogonal polynomials $\Psi$ that are functions of the random variables $\boldsymbol{\xi}$. For example, variable $u$ has the following spectral representation

$$
u(\mathbf{y}, \boldsymbol{\xi})=\sum_{k=0}^{\infty} \alpha_{k}(\mathbf{y}) \Psi_{k}(\boldsymbol{\xi})
$$

where $\Psi_{k}$ are the PC orthogonal polynomials and $\alpha_{k}$ the coefficients of the expansion. In practice, the series in Eq. (12) must be truncated to a finite number of terms $N$, which is determined by

$$
N+1=\frac{\left(n_{\xi}+n_{o}\right) !}{n_{\xi} ! n_{o} !}
$$

where $n_{\xi}$ is the dimension of the uncertainty vector $\boldsymbol{\xi}$ and $n_{o}$ is the order of the univariate polynomial expansion $\phi_{i}\left(\xi_{i}\right)$ from which the multivariate polynomials $\Psi_{k}(\boldsymbol{\xi})$ are obtained via tensorization, i.e.

$$
\Psi_{k}(\boldsymbol{\xi})=\prod_{i}^{n_{\xi}} \phi_{i}\left(\xi_{i}\right) .
$$

The polynomial basis $\phi_{i}\left(\xi_{i}\right)$ is chosen according to the Wiener-Askey scheme [47] in order to select orthogonal polynomials with respect to the probability density function $p_{\xi}(\boldsymbol{\xi})$ of the input. In this work, because a uniform distribution is considered, Legendre polynomials are employed. The orthogonality property can be advantageously used to compute the PC coefficients of the expansion $\alpha_{k}$ in a non-intrusive PC framework; this procedure is called NonIntrusive Spectral Projection (NISP) [44]. In fact, from the orthogonality property it directly follows that

$$
\alpha_{k}=\frac{\left\langle u(\mathbf{y}, \boldsymbol{\xi}), \Psi_{k}(\boldsymbol{\xi})\right\rangle}{\left\langle\Psi_{k}(\boldsymbol{\xi}), \Psi_{k}(\boldsymbol{\xi})\right\rangle} \quad \forall k
$$


The computation of the PC coefficients requires an integration of the polynomials that can be estimated with several approaches, among which quadrature formulae are chosen in this study. As a result, the solution of the deterministic problem is required for each quadrature point. Once the polynomial chaos and the associated $\alpha_{k}$ coefficients are computed, the mean value and the variance of the stochastic solution $u(\mathbf{x}, \boldsymbol{\xi})$ (Eq. (11)) are obtained leveraging the orthogonality of the polynomials $\Psi_{k}$ with respect to the probability function $p_{\xi}$ :

$$
\begin{aligned}
\left.\mu(u)\right|_{P C} & =\left\langle\sum_{k=0}^{N} \alpha_{k}(\mathbf{y}) \Psi_{k}(\boldsymbol{\xi})\right\rangle=\alpha_{0}(\mathbf{y}) \\
\left.\sigma^{2}(u)\right|_{P C} & =\left\langle\left(\sum_{k=0}^{N} \alpha_{k}(\mathbf{y}) \Psi_{k}(\boldsymbol{\xi})\right)^{2}\right\rangle-\alpha_{0}^{2}(\mathbf{y}) \\
& =\sum_{k=1}^{N} \alpha_{k}^{2}(\mathbf{y})\left\langle\Psi_{k}^{2}\right\rangle .
\end{aligned}
$$

Because a uniform distribution is considered also in the forward flight case, Legendre polynomials are employed in the PC expansion. The order of the expansion is retained from a convergence analysis which proved that a fourth-order polynomial is sufficient to accurately capture the statistics. The reconstruction of the statistics is based on the evaluation of the exact function for a set of samples $\boldsymbol{\xi}_{k}$ in the uncertain variables domain for each design vector $\overline{\boldsymbol{x}_{s}}$.

\section{Results}

As presented in the previous sections, different optimization problems are performed. A summary of the optimization cases studied in this work is given in Tab. 5. Letters DO and RO stand for the deterministic and robust optimizations, while numbers 1 and 2 refer to advancing and retreating 


\begin{tabular}{lllll}
\hline Case & Objective & $\begin{array}{l}\text { Equality } \\
\text { constraints }\end{array}$ & $\begin{array}{l}\text { Inequality } \\
\text { constraints }\end{array}$ & $\begin{array}{c}\text { Design } \\
\text { variables }\end{array}$ \\
\hline DO1 & $\min C_{D, 1}$ & $C_{L_{1}}=\overline{C_{L_{1}}}$ & $\left|C_{M_{1}}\right| \leq \overline{C_{M_{2}}}$ & $\boldsymbol{x}_{s}, \alpha$ \\
DO2 & $\min C_{D, 2}$ & $C_{L_{2}}=\overline{C_{L_{2}}}$ & $\left|C_{M_{2}}\right| \leq \overline{C_{M_{2}}}$ & $\boldsymbol{x}_{s}, \alpha$ \\
DO0 & $\min \sum_{i=1}^{2} w_{i} C_{D, i}$ & $C_{L_{1}}=\overline{C_{L_{1}}}$ & $\left|C_{M_{1}}\right| \leq \overline{C_{M_{1}}}$ & $\boldsymbol{x}_{s}, \alpha$ \\
& & $C_{L_{2}}=\overline{C_{L_{2}}}$ & $\left|C_{M_{2}}\right| \leq \overline{C_{M_{2}}}$ & \\
$\mathrm{RO} 1$ & $\max \mu\left(f_{1}\right)$ and $\min \left(\sigma^{2}\left(f_{1}\right)\right.$ & & $\left|C_{M_{1}}\right| \leq \overline{C_{M_{1}}}$ & $\boldsymbol{x}_{s}$ \\
$\mathrm{RO} 2$ & $\max \mu\left(f_{2}\right)$ and $\min \sigma^{2}\left(f_{2}\right)$ & & $\left|C_{M_{2}}\right| \leq \overline{C_{M_{2}}}$ & $\boldsymbol{x}_{s}$ \\
\hline
\end{tabular}

Table 5: Summary of optimization problems.

sides, respectively. The case DO0 represents the best trade off airfoil airfoil considering both retreating and advancing sides.

\subsection{Deterministic optimization}

The results of the deterministic optimization problems are: (i) airfoil DA1 with optimal drag coefficient on the advancing side, (ii) airfoil DA2 with optimal drag coefficient on the retreating side, and (iii) airfoil DA0 with optimal weighted sum of drag coefficients. These airfoils are presented in Fig. 10 and the characteristics of their geometry is reported in Tab. 6. It is possible to note that the optimal airfoil for the retreating side is highly cambered to cope with the higher angle of attack and it has a lower thickness-to-chord ratio to reduce the drag coefficient. The optimal airfoil of the advancing side generates positive lift owing to a slight camber and greater thickness. The slope of the camber line at the leading edge is controlled by the first coefficient of the parameterization thanks to the modification of the CST discussed in Section 3.1. Both in the overall airfoil geometry and in each specific parameter presented in Tab. 6 , it is possible to note that airfoil DA0 


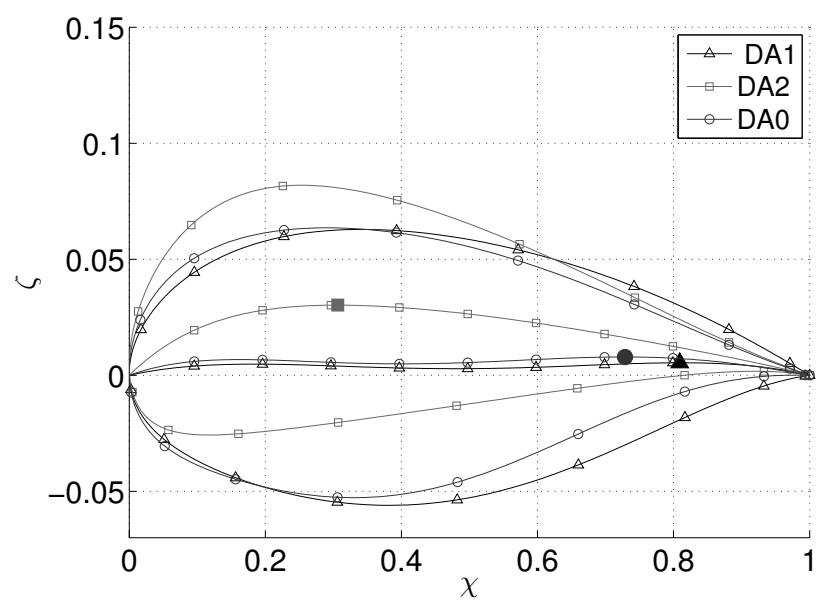

Figure 10: Optimal airfoils for optimization problem DA1, DA2 and DA0.

represents a trade-off solution between DA1 and DA2, although it appears more similar to the DA1 airfoil. This similarity is likely to be due to the choice of the weighting function.

\begin{tabular}{llll}
\hline & DA1 & DA2 & DA0 \\
\hline Maximum thickness & 0.1187 & 0.1049 & 0.1161 \\
Position of maximum thickness & 0.36 & 0.22 & 0.31 \\
Maximum camber & 0.0054 & 0.0303 & 0.0079 \\
Position of max camber & 0.81 & 0.31 & 0.73 \\
First design variable $\left(A_{0}^{c}\right)$ & 0.065 & 0.289 & 0.107 \\
Camber slope at LE $[\mathrm{deg}]$ & 3.704 & 16.098 & 6.108 \\
\hline
\end{tabular}

Table 6: Characteristics of the optimal airfoils (for unitary chord).

With regard to performance, the drag coefficients of the optimal airfoils are presented in Tab. 7. The drag coefficient are expressed in drag counts, where one drag count is equal to 0.0001 . The gain with respect to the baseline NACA 23012 airfoil are presented in Tab. 8. The DA1 airfoil and the DA2 


\begin{tabular}{lllllll}
\hline & $\begin{array}{l}C_{D_{1}} \\
{[\text { counts }]}\end{array}$ & $\begin{array}{l}\left.\alpha_{1}\right|_{\overline{L_{1}}} \\
{[\mathrm{deg}]}\end{array}$ & $\begin{array}{l}C_{M_{1}} \\
{[-]}\end{array}$ & $\begin{array}{l}C_{D_{2}} \\
{[\text { counts }]}\end{array}$ & $\begin{array}{l}\left.\alpha_{2}\right|_{\overline{L_{2}}} \\
{[\mathrm{deg}]}\end{array}$ & $\begin{array}{l}C_{M_{2}} \\
{[-]}\end{array}$ \\
\hline DA0 & 0.8947 & -1.059 & 0.0463 & 177.8 & 11.1 & -0.0108 \\
DA1 & 0.6927 & -1.002 & 0.0459 & - & - & - \\
DA2 & 191.99 & -3.101 & 0.1047 & 126.0 & 9.27 & -0.0393 \\
\hline
\end{tabular}

Table 7: Performance of the optimal airfoils: performance of DA1 in the retreating side is not reported because DA1 airfoil cannot satisfy lift constraint (see Fig. 11b).

\begin{tabular}{ccc|cc}
\hline & $\begin{array}{c}\text { Percentage gain } \\
\text { side 1 }\end{array}$ & $\begin{array}{c}\text { Percentage gain } \\
\text { side 2 }\end{array}$ & $\begin{array}{c}\text { Relative gain } \\
\text { side 1 }\end{array}$ & $\begin{array}{c}\text { Relative gain } \\
\text { side 2 }\end{array}$ \\
\hline DA0 & $+99 \%$ & $+7 \%$ & $1 / 97.2$ & $1 / 1.08$ \\
DA1 & $+99 \%$ & - & $1 / 125$ & - \\
DA2 & - & $+34 \%$ & - & $1 / 1.52$ \\
\hline
\end{tabular}

Table 8: Gain of the optimal airfoils with respect to the baseline NACA 23012 airfoil: gain of DA1 in the retreating side and gain of DA2 in the advancing side are not reported because these airfoils do not satisfy respectively the lift and moment constraints.

airfoil significantly improve the performance of the advancing and retreating sides, respectively. This was expected for a deterministic optimization that starts from a baseline airfoil that has been used extensively on rotorcraft, as for the Bo-105 helicopter, but that was not specifically developed for rotorcraft flow conditions.

Airfoil DA1 is an airfoil which reduces the drag coefficient by smoothing curvature variations of the upper and lower sides, yielding a shock-free flowfield (see the Mach number contour in Fig. 11a). However, on the retreating side, the DA1 airfoil cannot satisfy the lift constraint (as presented 


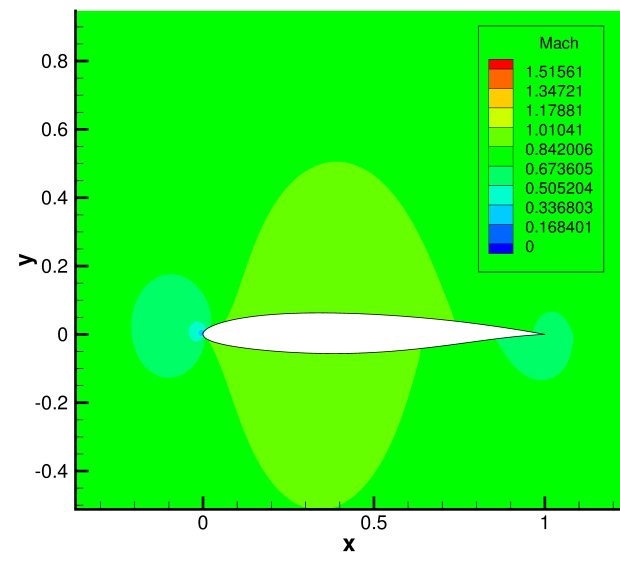

(a) Mach number contour in the advancing side for airfoil DA1.

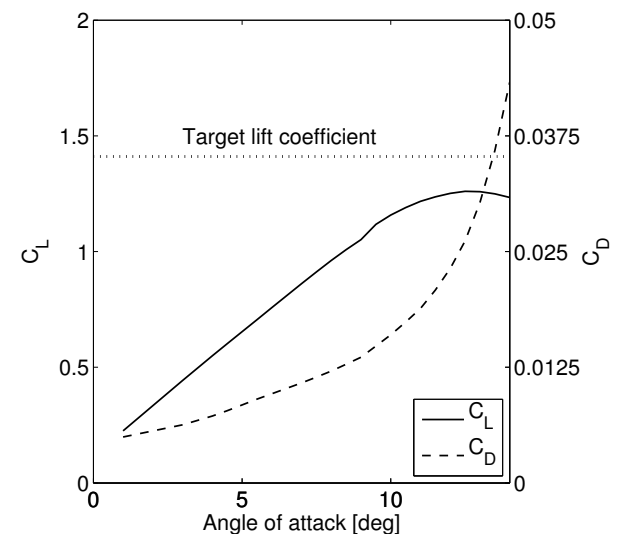

(b) Lift and drag coefficient in the retreating side for airfoil DA1.

Figure 11: Analysis of optimal DA1 airfoil.

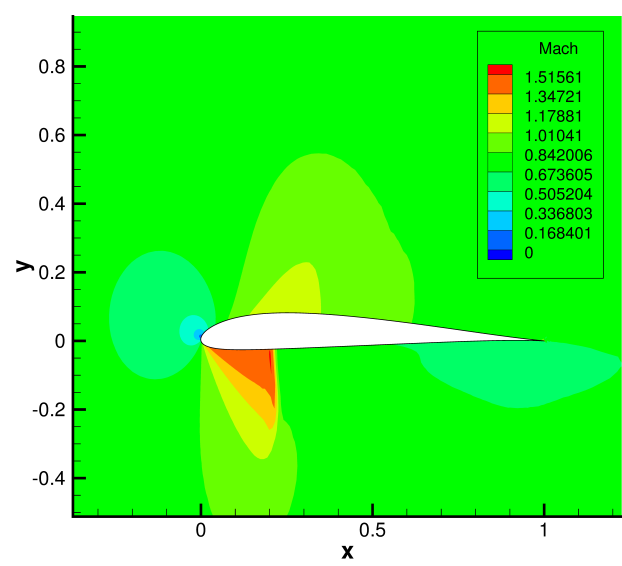

(a) Mach number contour in the advancing side for airfoil DA2.
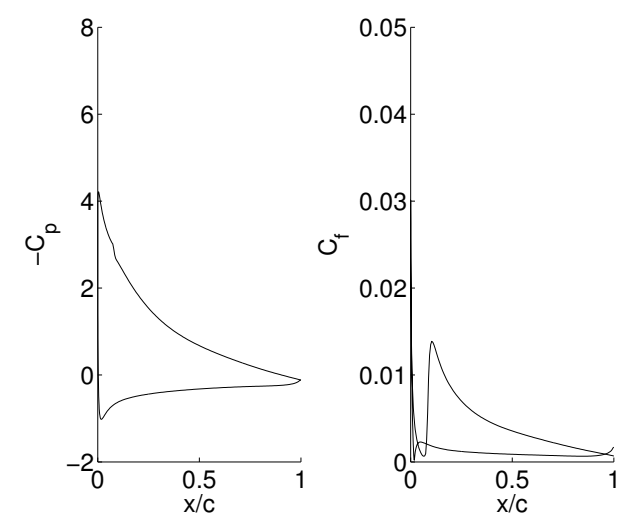

(b) Pressure and friction coefficient in the retreating side for airfoil DA2.

Figure 12: Analysis of optimal DA2 airfoil.

in Fig. 11b), therefore its drag coefficient is not reported in Tab. 7 and its gain is not present in Tab. 8. On the other side, airfoil DA2 reduces the drag coefficient of the NACA 23012 airfoil by reducing the peak friction coefficient and by postponing boundary layer transition, as presented in Fig. 12b. 


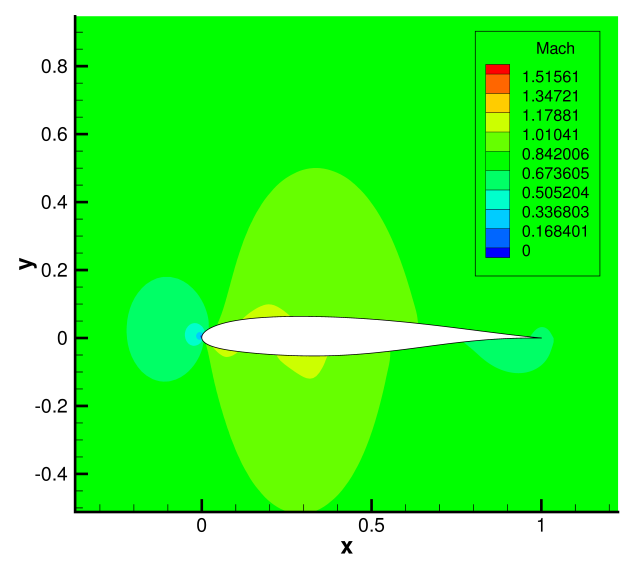

(a) Mach number contour in the advancing side for airfoil DA0.
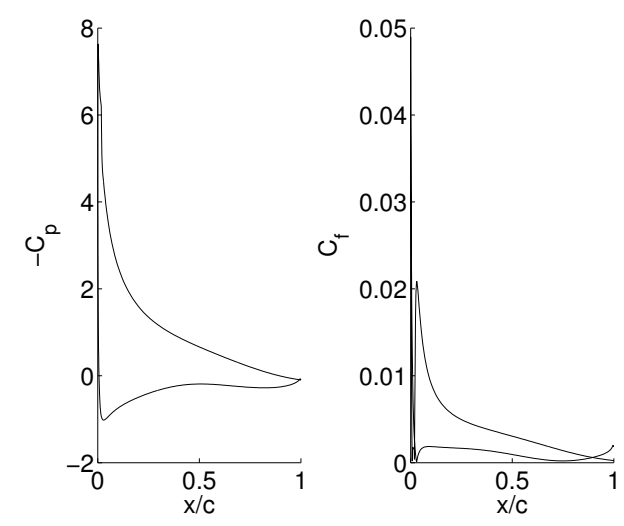

(b) Pressure and friction coefficient in the retreating side for airfoil DA0.

Figure 13: Analysis of optimal DA0 airfoil.

However, on the advancing side, the performance of such an airfoil is very poor and a strong shock wave is present on the lower side (see Fig. 12a). The trade-off airfoil DO0 has a significant gain on the advancing side, because it is more similar to the DA1 airfoil. As a matter of fact, a shock-free flowfield is obtained with this airfoil (Fig. 13a), whereas on the retreating side, an earlier transition to a turbulent boundary layer is present, and so a higher friction coefficient (Fig. 13b).

\subsection{Comparison with robust optimization}

Two different robust optimization problems are performed, the first one for the advancing side (problem RO1) and the second one for the retreating side (RO2). The Pareto front for the case of the advancing side is presented in Fig. 14a where the two objective functions are the mean value and the variance of the lift-to-drag ratio. Please note that in the figure the mean value and the variance are obtained with a scaling factor of 1/10 acting on $f_{1}$, because the value of $f_{1}=C_{L} / C_{D}$ is very large due to the inviscid estimate 


\begin{tabular}{lccccc}
\hline Airfoil & $\begin{array}{c}C_{D, 1} \mid \overline{C_{L_{1}}} \\
{[\text { counts }]}\end{array}$ & $\begin{array}{c}\left.\alpha_{1}\right|_{\overline{C_{1}}} \\
{[\mathrm{deg}]}\end{array}$ & $\begin{array}{c}\mu_{C_{D, 1}} \\
{[\text { counts }]}\end{array}$ & $\begin{array}{c}\sigma_{C_{D, 1}}^{2} \\
{\left[\text { counts }^{2}\right]}\end{array}$ & $\begin{array}{c}(\sigma / \mu)_{C_{D, 1}} \\
{[-]}\end{array}$ \\
\hline DA0 & 0.895 & -1.059 & 5.26 & 65.75 & 1.54 \\
DA1 & 0.693 & -1.002 & 2.16 & 11.12 & 1.54 \\
RA1 & 0.898 & -1.510 & 2.03 & 5.668 & 1.17 \\
\hline
\end{tabular}

Table 9: Drag coefficient of the deterministic airfoil minimizing $C_{D_{1}}$ (DA1), the trade-off airfoil (DA0) and the airfoil selected from the robust front in the advancing side (RA1).

of the drag coefficient which is very small for shock-free optimal airfoils (on the order of a drag count). The front presents very robust solution that, however, comes at the expense of poor performance. In the higher part of the front, solutions with high mean value are found.

To compare the results obtained from the Pareto front in RO1 and the deterministic result DA1, a solution or a group of solutions from the front

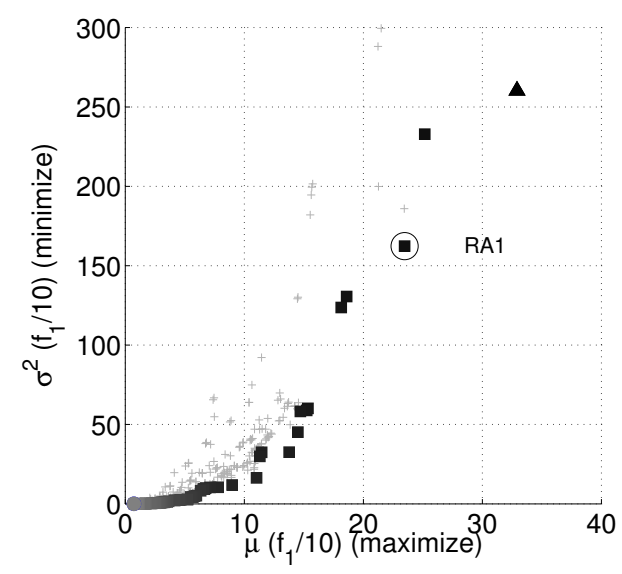

(a) Pareto front. The triangle indicates the airfoil with maximum $\mu$.

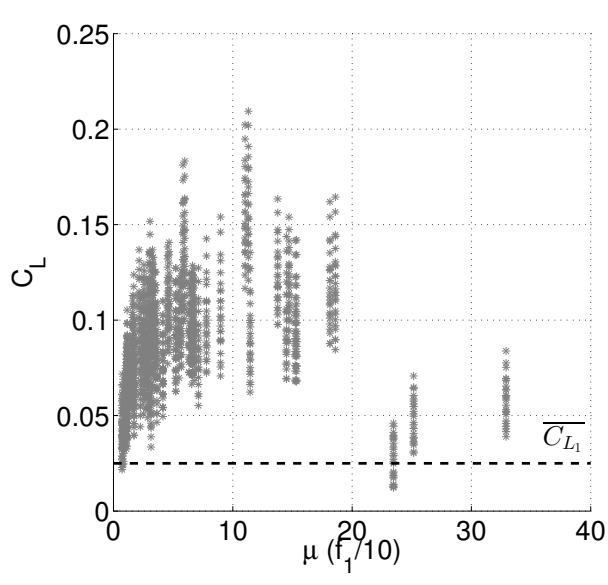

(b) Lift coefficient in the samples used in RO1 for the airfoils of the Pareto front.

Figure 14: Results of the robust optimization for the advancing side (RO1) . 


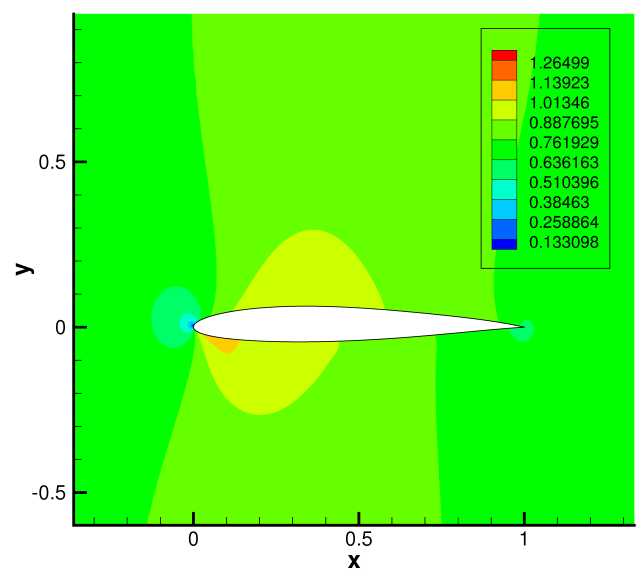

Figure 15: Mach number contour of RA1 airfoil at $\left.\alpha_{1}\right|_{\overline{C_{L_{1}}}}=-1.51 \mathrm{deg}$.

in Fig. 14a should be selected. To do this, the target lift coefficient $\overline{C_{L_{1}}}$ is compared to the lift coefficients obtained in the samples used for the reconstruction of the mean value and variance in the UQ loop. The lift coefficients for the solutions of the front are plotted in Fig. 14b. From this set, airfoil RA1 is selected, which is the airfoil that exhibits a lift coefficient equal to the target value inside the uncertainty range considered and a high mean value of the lift-to-drag ratio. Airfoil RA1 is also highlighted in Fig. 14a. The value of the angle of attack for which the lift coefficient of airfoil RA1 is equal to $\overline{C_{L_{1}}}$ is called $\left.\alpha_{1}\right|_{\overline{C_{L_{1}}}}$ and it is equal to -1.51 degrees at the Mach number used for the deterministic optimization DO1. As presented in Tab. 9, in this condition the performance of RA1 airfoil is similar to that of the other two optimized in a deterministic way, DA1 and DA0, since even this airfoil does not present extended region of supersonic flow and shock waves (see Fig. 15). However, if an uncertainty band is considered around the condition ensuring trim requirement, both the mean value and the variance of the drag coefficient for airfoil RA1 are smaller than the values of airfoil DA1 (see Tab. 9). The table also presents the coefficient of variation, defined as the ratio of the 


\begin{tabular}{lcccccc}
\hline Airfoil & $\mu_{C_{L, 1}}$ & $\sigma_{C_{L, 1}}^{2}$ & $(\sigma / \mu)_{C_{L, 1}}$ & $\mu_{C_{M, 1}}$ & $\sigma_{C_{M, 1}}^{2}$ & $(\sigma / \mu)_{C_{M, 1}}$ \\
\hline DA0 & 0.0257 & $4.52 \mathrm{e}-05$ & 0.26132 & 0.0459 & $9.87 \mathrm{e}-07$ & 0.0217 \\
DA1 & 0.0211 & $4.04 \mathrm{e}-05$ & 0.30151 & 0.0457 & $1.81 \mathrm{e}-06$ & 0.0294 \\
RA1 & 0.0253 & $8.20 \mathrm{e}-05$ & 0.35816 & 0.0688 & $1.47 \mathrm{e}-06$ & 0.0176 \\
\hline
\end{tabular}

Table 10: Lift and moment coefficient of the deterministic airfoil minimizing $C_{D_{1}}$ (DA1), the trade-off airfoil (DA0) and the airfoil selected from the robust front in the advancing side (RA1).

standard deviation $\sigma$ to the mean value $\mu$, to appreciate the dispersion with respect to the mean value.

To complete the analysis, Table 10 presents the mean value and variance of the lift and moment coefficients for airfoil RA1 and airfoil DA1. In the table, it is interesting to note that the mean value of airfoil RA1 is closer to

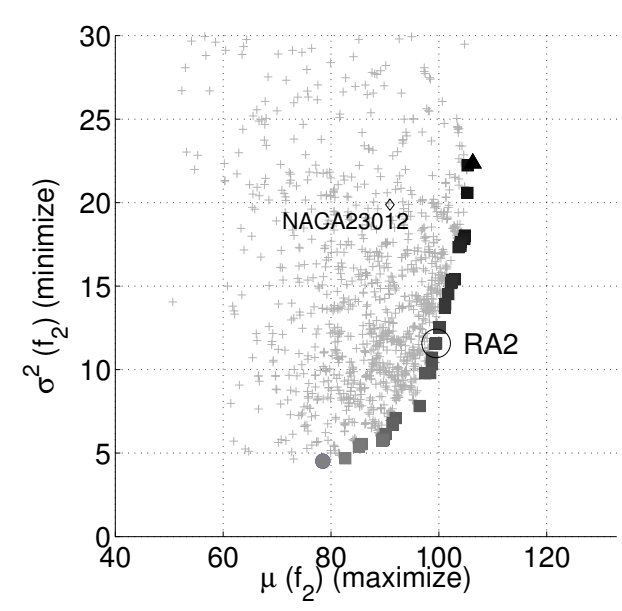

(a) Pareto front.The triangle indicates the airfoil with maximum $\mu$.

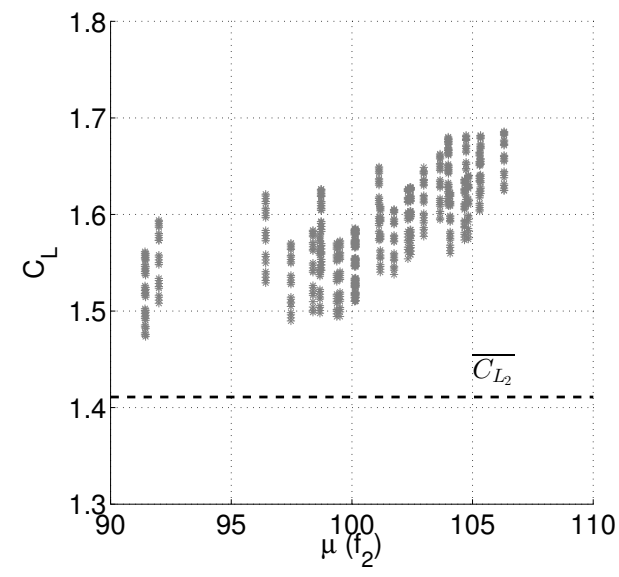

(b) Lift coefficient in the samples used in $\mathrm{RO} 2$ for the airfoils of the Pareto front.

Figure 16: Results of the robust optimization for the retreating side (RO2) . 
the target value. It is noted that the mean value of the drag coefficient, lift coefficient, and moment coefficient are obtained with the fourth-order PC expansion, which has been assessed within a convergence study, not reported here for brevity [32]. Despite the presence of shock waves in this range of conditions, the integral values remain smooth throughout the stochastic space and a fourth-order polynomial provides sufficiently accurate estimates of the statistics.

The same analysis can be performed for the Pareto front of the retreating side. Figure 16a presents the optimal set of solutions obtained from the robust optimization. As emerged from the results in [19] for the hovering case and in the advancing side, interesting solutions from the front are typically those with high mean performance. A possible criterion for decision making in the post-processing of the front is then the selection of non-dominated solutions with mean value higher than the value of the reference airfoil. This criterion helps removing the solutions with poor performance, but it still retains solutions with variance lower than the reference result thanks to the quality of the Pareto front.

For this subset of airfoils, the comparison between the robust front and the deterministic airfoil is performed in the same way as presented for the advancing side. Figure 16b shows the lift coefficient of the samples used in RO2 with a mean value higher than the reference value. In this case, every airfoil in the optimal set provides a lift coefficient that is greater than the target value $\overline{C_{L_{2}}}$. Thus, to select an airfoil from the front for comparison, airfoil RA2 has been chosen as a trade-off between the two objectives.

For this airfoil, an angle of attack equal to $10.66 \mathrm{deg}$ is needed to satisfy the trim condition. By applying the same uncertainty range used in the robust optimization case, it is possible to compute the mean value and the 

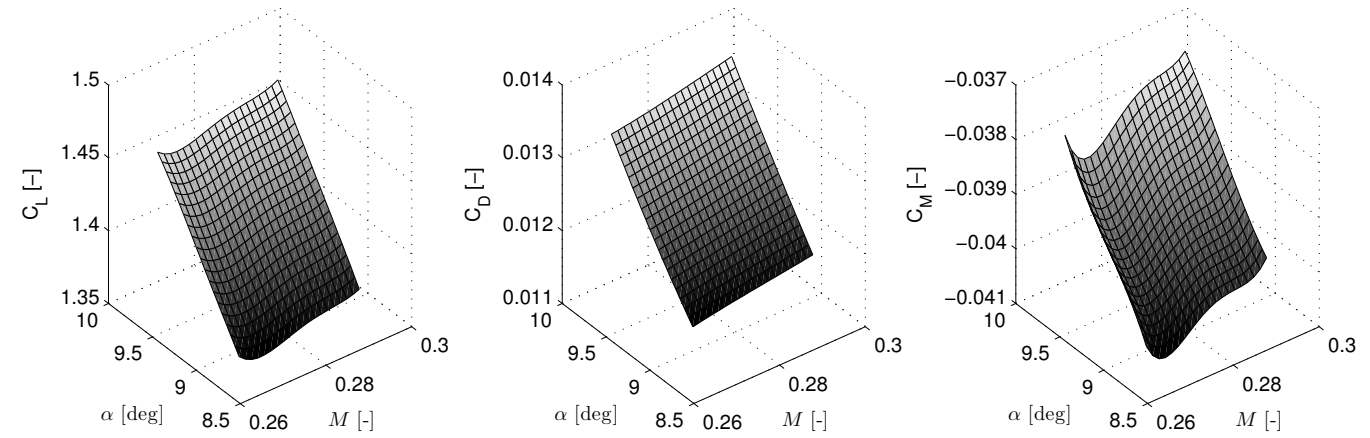

Figure 17: Load of DA2 in the uncertainty band around $\left.\alpha_{1}\right|_{\overline{C_{1}}}$.

\begin{tabular}{cccccc}
\hline Airfoil & $\begin{array}{c}\left.C_{D, 2}\right|_{\overline{C_{L}}} \\
{[\text { counts }]}\end{array}$ & $\begin{array}{c}\left.\alpha_{1}\right|_{\overline{L_{2}}} \\
{[\mathrm{deg}]}\end{array}$ & $\begin{array}{c}\mu_{C_{D, 2}} \\
{[\text { counts }]}\end{array}$ & $\begin{array}{c}\sigma_{C_{D, 2}}^{2} \\
{\left[\text { counts }^{2}\right]}\end{array}$ & $\begin{array}{c}(\sigma / \mu)_{C_{D, 2}} \\
{[-]}\end{array}$ \\
\hline DA0 & 177.8 & 11.13 & 178.4 & 91.32 & 0.0536 \\
DA2 & 126.0 & 9.27 & 126.2 & 28.40 & 0.0422 \\
RA2 & 148.1 & 10.66 & 148.4 & 33.83 & 0.0392 \\
\hline
\end{tabular}

Table 11: Drag coefficient of the deterministic airfoil minimizing $C_{D_{2}}$ (DA2), the trade-off airfoil (DA0) and the airfoil selected from the robust front in the retreating side (RA2).

variance of the drag coefficient of RA2. These values are higher with respect to the value computed for airfoil DA2 (see Tab. 11). In fact, the optimal deterministic airfoil has a very low value of drag coefficient because it satisfies the lift constraint at a very low angle of attack (9.27 deg) thanks to the highly cambered mean line. However, airfoil DA2 cannot satisfy the moment coefficient constraint in the uncertainty range. As presented in Fig. 17, the moment coefficient exceeds the threshold value $\overline{C_{M_{2}}}$. On the contrary, the robust optimization formulation guarantees that the optimal airfoils would not incur moment penalties throughout the uncertainty range.

Finally, the discrepancy in the performance of airfoil DA2 and airfoil RA2 is due to the fact that the lift coefficient of the robust airfoils are significantly 


\begin{tabular}{lcccccc}
\hline Airfoil & $\mu_{C_{L, 2}}$ & $\sigma_{C_{L, 2}}^{2}$ & $(\sigma / \mu)_{C_{L, 2}}$ & $\mu_{C_{M, 2}}$ & $\sigma_{C_{M, 2}}^{2}$ & $(\sigma / \mu)_{C_{M, 2}}$ \\
\hline DA0 & 1.3998 & $8.37 \mathrm{e}-04$ & 0.207 & $-1.069 \mathrm{e}-02$ & $3.84 \mathrm{e}-06$ & -0.18 \\
DA2 & 1.4113 & $8.67 \mathrm{e}-04$ & 0.021 & $-3.924 \mathrm{e}-02$ & $6.51 \mathrm{e}-07$ & -0.02 \\
RA2 & 1.4016 & $6.24 \mathrm{e}-04$ & 0.018 & $-1.383 \mathrm{e}-02$ & $7.68 \mathrm{e}-06$ & -0.20 \\
\hline
\end{tabular}

Table 12: Lift and moment coefficient of the deterministic airfoil minimizing $C_{D_{2}}$ (DA2), the trade-off airfoil (DA0) and the airfoil selected from the robust front in the retreating side (RA2).

greater than the target lift coefficient. Thus, the deterministic optimization and the robust optimization are essentially exploring different regions of the design space, according to the specific objectives. To some extent, a fair comparison between the two methods is meaningful if the two methods share the "same" objectives. This means, for example, that for this specific case the target lift coefficient should be higher for the comparison with the robust airfoils. Another possibility is to define the nominal condition for the robust case starting from the deterministic results. This would at least ensure the presence of the deterministic airfoil in the robust population (as long as it satisfies the constraints). This last strategy appears more straightforward, and the results of its application are reported in another paper [48].

\section{Verification of design along the full azimuth angle range}

Up to this point, the performance of the optimal airfoils is considered only for the advancing and retreating side conditions. However, the blade section encounters different operating conditions over the period of rotation of the blade and an assessment of the performance with the azimuth angle is appropriate. To do this, the variation of the angle of attack, Mach number, and Reynolds number with the azimuth angle must be computed. This 
computation is based on the varying freestream Mach number and angle of attack plotted in Fig. 1. The variation of angle of attack and Mach number presented in the figure are described by means of the following time law

$$
\begin{aligned}
M(t) & =M_{\infty}(1+\lambda \sin (\Omega t)) \\
\alpha(t) & =\alpha_{0}+\sum_{i=1}^{n_{h}} \Delta \alpha_{i} \sin \left(i \Omega t+\bar{\psi}_{i}\right), \quad n_{h}=4
\end{aligned}
$$

where $\Omega$ is the blade $1 /$ rev frequency (such that the azimuth angle $\psi$ satisfies $\psi=\Omega t), M_{\infty}$ is the freestream Mach number, and $\lambda$ is the percentage variation of Mach number. The angle of attack is described by means of a sine series, where $\alpha_{0}$ is the mean angle of attack, $\Delta \alpha_{i}$ is the angle of attack variation of the $i$-th term in the series and $\bar{\psi}_{i}$ is the $i$-th phase angle. This

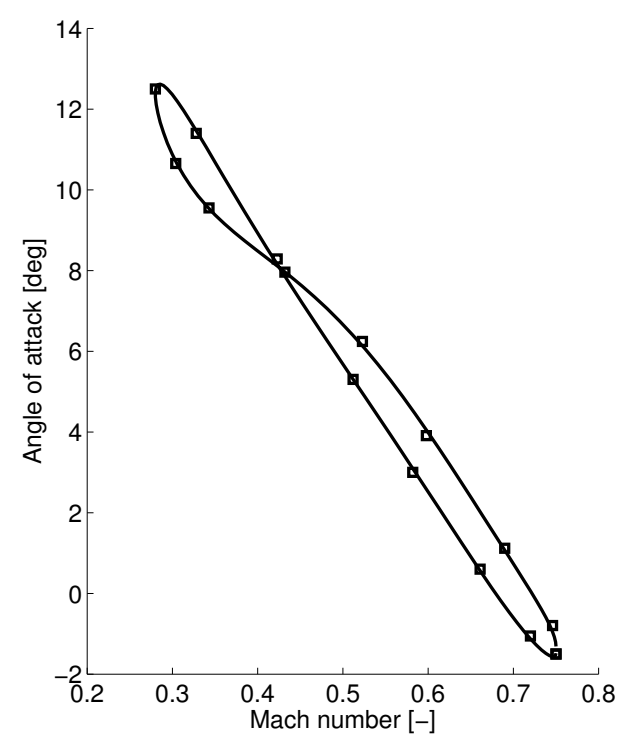

(a) Cycle $\alpha-M$ : reference cycle from Fig. 1 (line) and samples from approximation in Eq. (15) (squares).
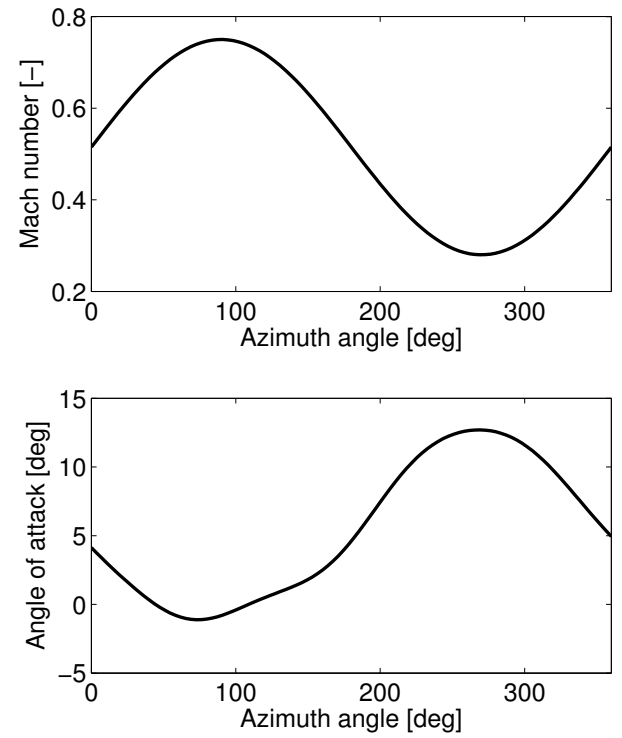

(b) Corresponding time varying Mach number (top) and angle of attack (bottom).

Figure 18: Time varying Mach number and angle of attack encountered by the blade element (Eq. (15)). 


\begin{tabular}{ccccccccccc}
\hline $\begin{array}{c}M_{\infty} \\
{[-]}\end{array}$ & $\lambda$ & $\alpha_{0}$ & $\Delta \alpha_{1}$ & $\phi_{1}$ & $\Delta \alpha_{2}$ & $\phi_{2}$ & $\Delta \alpha_{3}$ & $\phi_{3}$ & $\Delta \alpha_{4}$ & $\phi_{4}$ \\
\hline-$]$ & {$[\mathrm{deg}]$} & {$[\mathrm{deg}]$} & {$[\mathrm{deg}]$} & {$[\mathrm{deg}]$} & {$[\mathrm{deg}]$} & {$[\mathrm{deg}]$} & {$[\mathrm{deg}]$} & {$[\mathrm{deg}]$} & {$[\mathrm{deg}]$} \\
\hline 0.515 & 0.45 & 5.36 & 6.84 & -173.8 & 0.67 & -86.0 & 0.28 & 112.3 & 0.2 & -22.5 \\
\hline
\end{tabular}

Table 13: Variables of time-varying angle of attack and Mach number in Eq. (15).

structure of the variations has been obtained with a convergence analysis based on a sine series with increasing number of terms. Figure 18a shows the reference $\alpha-M$ cycle , while Fig. 18b shows the variation of the angle of attack and Mach number with the azimuth. The values of the variables in Eq. (15) are presented in Tab. 13. They directly correspond to the extrema used in the optimization loop: (i) a maximum value of the angle of attack equal to $12.5 \mathrm{deg}$ at a Mach number of 0.28 and (ii) a minimum value equal to -1.5 deg at a Mach number equal to 0.75 (see Tab. 1).

With the laws of Eq. (15) and values of Tab. 13 the baseline NACA 23012 develops the trim loads required. However, for the optimal airfoils, new values of the amplitude of the sine terms describing the angle of attack must be computed, because the target lift coefficient is obtained on both sides at a different angles of attack. In other words, the amplitude of the time-varying angle of attack presented in Fig. 18b must be corrected for each optimal shape. In general, the azimuth variation of the angle of attack is caused by the blade cyclic pitch control, the induced velocity of the rotor, the blade dynamics, and aeroelastic deformations. Thus, as a first approximation, it is possible to associate the $2 / \mathrm{rev}, 3 / \mathrm{rev}$ and $4 / \mathrm{rev}$ contributions in Eq. (15) to the nonlinear contributions depending on the aerodynamic effects of vortices, wakes and the blade dynamics. These contributions are essentially a function of the lift produced by the blade, which remains unchanged for the optimal airfoils. However, the first sine term $\Delta \alpha_{1}(1 / \mathrm{rev})$ is directly related to the 


\begin{tabular}{lccccc}
\hline & DA0 & DA1 & DA2 & RA1 & RA2 \\
\hline$\alpha_{0}[\mathrm{deg}]$ & 5.04 & 5.75 & 3.09 & 5.49 & 4.58 \\
$\Delta \alpha_{1}[\mathrm{deg}]$ & 5.88 & 6.51 & 5.97 & 6.76 & 5.87 \\
\hline
\end{tabular}

Table 14: Variables of time-varying angle of attack (Eq. (15)) for optimal shapes.

cyclic pitch control and can be adjusted to maintain the trim requirement. So, only this term is updated for the optimized airfoils, as shown in Tab. 14.

Using the same procedure shown in section 2, steady state computation are performed at eight station along the azimuth angle. The aerodynamic coefficients for the deterministic optimal airfoils (DA0, DA1, DA2) are presented in Fig. 19. It is possible to note that the cycle of the lift coefficient is similar for the three airfoils owing to the equality constraint on the lift coefficient used in the optimization loop. Please recall that airfoil DA1 is not capable of satisfying the trim constraint in the off-design condition (i.e. the retreating side), as emerged from Fig. 11b.

The average of the drag coefficient over the azimuth angle $\operatorname{avg}\left(C_{D}\right)$ is then computed and the results are summarised in Tab. 15. The table also presents the results for the airfoils selected from the robust Pareto front. i.e. airfoil RA1 and airfoil RA2. The associated cycles of the aerodynamic coefficients is presented in Fig. 20. As expected, airfoil DA0 provides the best average drag coefficient over the azimuth angle. The penalty of airfoil DA1 in the off-design condition (i.e. the retreating side) is higher than the penalty 

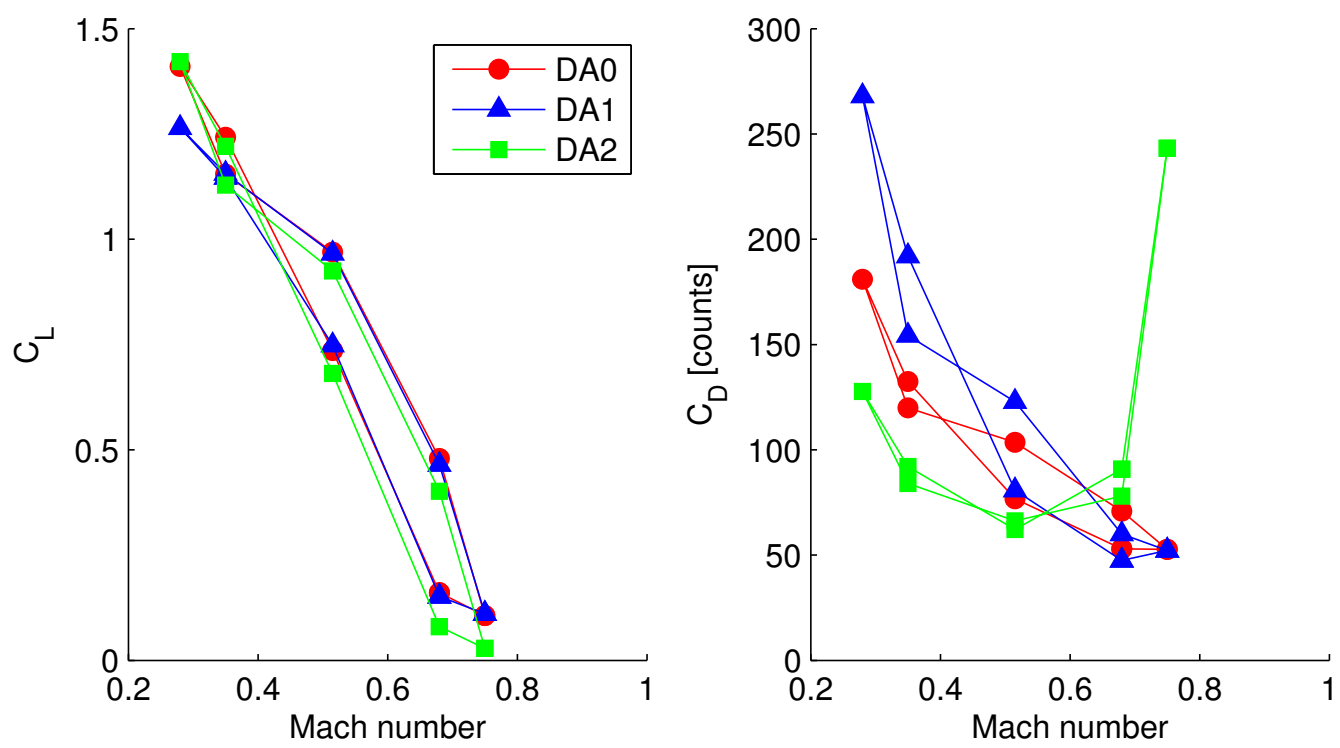

Figure 19: Lift and drag coefficient with azimuth for deterministic optimal airfoils.

\begin{tabular}{lccccc}
\hline Airfoil & DA0 & DA1 & DA2 & RA1 & RA2 \\
$\operatorname{avg}\left(C_{D}\right)[$ counts $]$ & 98.76 & 122.22 & 105.5 & 118.84 & 107.71 \\
\hline
\end{tabular}

Table 15: Average drag coefficient for optimal airfoils in forward flight.

suffered by airfoil DA2 in the off-design conditions. As a result, airfoil DA2 has a better average value. Airfoil RA2 has a small penalty with respect to its deterministic counterpart and it also presents a fairly symmetrical cycle. Airfoil RA1 on the other hand has a better average value than the corresponding deterministic airfoil.

\section{Conclusions}

A methodology for robust optimization has been developed to tackle the problem of designing airfoils in forward flight. Robust optimization requires the coupling of the uncertainty quantification method and the optimization algorithm. This coupling increases the computational demand, especially in 

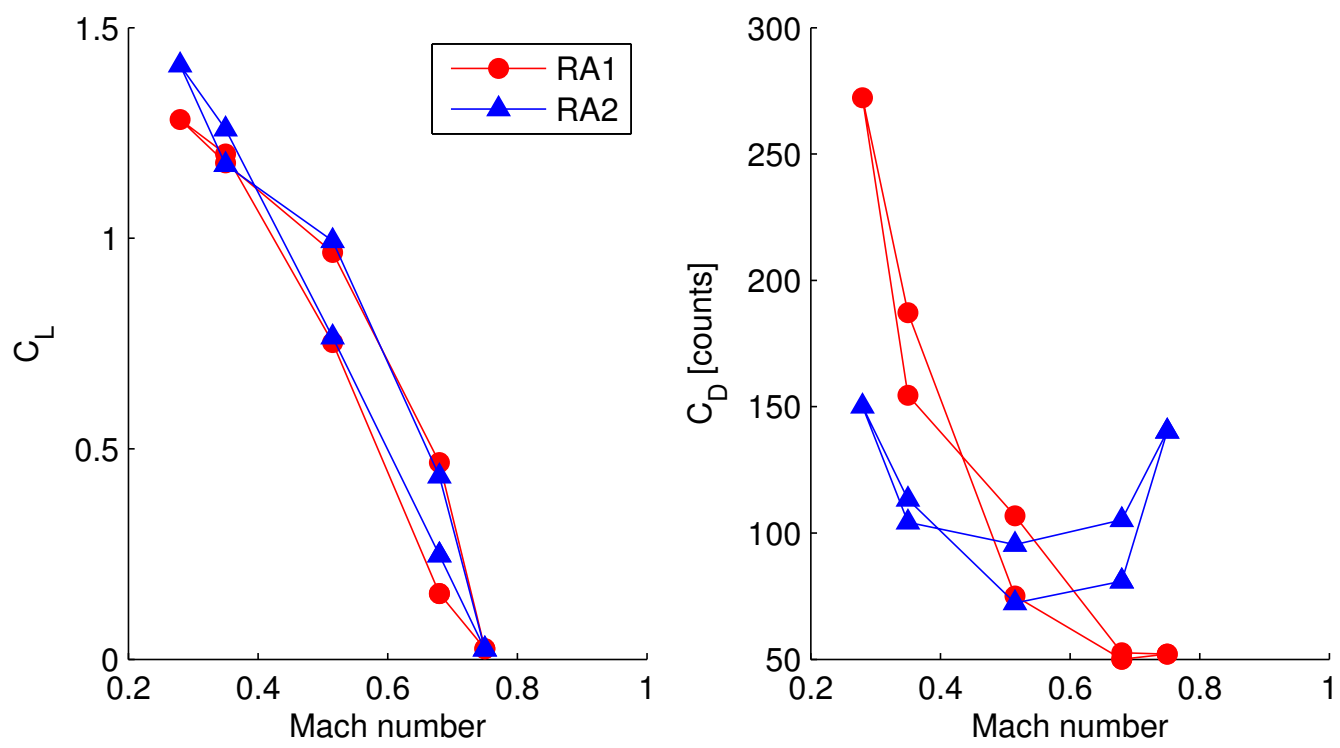

Figure 20: Lift and drag coefficient with azimuth for robust optimal airfoils.

aerodynamic applications, where a single function evaluation may be very expensive.

To obtain comparable results through robust and deterministic optimizations it has been necessary to consider fairly different problem set-up.

Deterministic results have proven how different the optimal airfoils for advancing blade is compared to the retreating case. Comparison with the robust approaches have shown similarities and differences between the use of the drag coefficient as a deterministic objective and the employment of the lift-to-drag ratio in a robust context. In general, the robust approach has shown the capability to define airfoils that have a mean performance close to the one obtained through a deterministic approach. At the same time, the choice of low variance airfoils though the application of robust approaches ensures the possibility to obtain airfoils that perform adequately without violating the constraints also in off-design condition. This is a characteristic that should be considered fairly important when designing airfoils for ro- 
torcraft, where a significant variability of the operating conditions could be expected.

The identification of the optimal airfoil directly through a robust formulation as the one proposed here may result cumbersome, given the difficulty in the identification of an optimal airfoil with an uncertainty band across the required lift coefficient. So, a sequential strategy that considers first the application of the deterministic optimization, and then that of the robust optimization starting from the optimal deterministic airfoils should be considered the best approach to arrive to airfoils with good performances that suffer of a limited degradation in off-design conditions.

\section{References}

[1] W. Johnson, Rotorcraft Aeromechanics, Cambridge University Press, New York, 2013.

[2] M. Drela, Pros and cons of airfoil optimization, in: D. Caughey, M. Hafez (Eds.), Frontiers of Computational Fluid Dynamics, World Scientific Publishing, 1998, pp. 363-382.

[3] A. Datta, I. Chopra, Prediction of the UH-60A Main Rotor Structural Loads Using Computational Fluid Dynamics/Comprehensive Analysis Coupling, Journal of the American Helicopter Society 53 (4) (2008) 351. doi:10.4050/JAHS.53.351.

[4] M. Bhagwat, H. Ormiston, R.and Saberi, H. Xin, Application of computational fluid dynamics/computational structural dynamics coupling for analysis of rotorcraft airloads and blade loads in maneuvering flight, Journal of the American Helicopter Society 57 (3). 
[5] A. Antoniadis, D. Drikakis, G. Zhong, B.and Barakos, R. Steijl, M. Biava, L. Vigevano, A. Brocklehurst, O. Boelens, M. Dietz, M. Embacher, W. Khier, Assessment of cfd methods against experimental ow measurements for helicopter flows, Aerospace Science and Technology 19 (1) (2012) 86-100.

[6] A. Le Pape, P. Baumier, Numerical optimization of helicopter rotor aerodynamic performance in hover, Aerospace Science and Technology 9 (3) (2005) 191-201.

[7] H. Sun, S. Lee, Response surface approach to aerodynamic optimization design for helicopter rotor blade, International Journal for Numerical Methods in Engineering 64 (2005) 125-142.

[8] C. A. Tatossian, S. K. Nadarajah, P. Castonguay, Aerodynamic shape optimization of hovering rotor blades using a non-linear frequency domain approach, Computers and Fluids 51 (1) (2001) 1-15.

[9] H. Farrokhfal, A. R. Pishevar, Aerodynamic shape optimization of hovering rotor blades using a coupled free wake-CFD and adjoint method, Aerospace Science and Technology 1 (2012) 1-10.

[10] K. Collins, L. Sankar, D. Mavris, Application of low- and high-fidelity simulation tools to helicopter rotor blade optimization, Journal of the American Helicopter Society 58 (4) (2013) 1-10.

[11] A. Mishra, K. Mani, D. Mavriplis, J. Sitaraman, Time dependent adjoint-based optimization for coupled fluid-structure problems, Journal of Computational Physics 292 (2015) 253-271. doi:10.1016/j.jcp.2015.03.010. 
[12] D. Zingg, S. Elias, Aerodynamic Optimization Under a Range of Operating Conditions, AIAA Journal 44 (11) (2006) 2787-2792. doi:10.2514/1.23658.

[13] W. G. Bousman, Airfoil Design and Rotorcraft Performance., in: American Helicopter Society 58th Annual Forum, Montreal, Canada, 2002.

[14] J. G. Leishman, Principles of helicopter aerodynamics, Cambridge University Press, 2006.

[15] S. K. Nadarajah, A. Jameson, Optimum Shape Design for Unsteady Flows with Time-Accurate Continuous and Discrete Adjoint Method, AIAA Journal 45 (7) (2007) 1478-1491.

[16] D. Fanjoy, W. A. Crossley, Aerodynamic shape design for rotor airfoils via genetic algorithm, Journal of the American Helicopter Society 43 (3) (1998) 263-270.

[17] A. Morris, C. Allen, T. Rendall, Aerodynamic optimisation of hovering helicopter rotors using efficient and flexible shape parameterisation, in: 26th AIAA Applied Aerodynamics Conference, 2008.

[18] A. Massaro, E. Benini, Multi-Objective Optimization of Helicopter Airfoils Using Surrogate-Assisted Memetic Algorithms, Journal of Aircraft 49 (2) (2012) 375-383.

[19] F. Fusi, A. Guardone, G. Quaranta, P. M. Congedo, Multi-fidelity physics-based method for robust optimization with application to a hovering rotor airfoil, AIAA Journal 53 (11) (2015) 3448-3465.

[20] R. L. Peterson, Full-scale hingeless rotor performance and loads, TM 110356, NASA (1995). 
[21] B. G. van der Wall, $2^{\text {nd }}$ HHC Aeroacoustic Rotor Test (HART II) - Part I: Test Documentation., Braunschweig, Germany, 2003.

[22] D. J. Gosselin, 2d dynamic stall simulations with time-varying freestream representative of helicopter flight, Ph.D. thesis, OttawaCarleton Institute for Mechanical \& Aerospace Engineering (2014).

[23] D. Favier, A. Agnes, C. Barbi, C. Maresca, A new simulation of airfoil dynamic stall due to velocity and incidence fluctuations, in: 19th AIAA, Fluid Dynamics, Plasma Dynamics and Lasers Conference, American Institute of Aeronautics and Astronautics, 1987.

[24] D. Favier, C. Maresca, C. Barbi, Unsteady aerodynamics of an airfoil in combined translation/pitch oscillations below and through stall, in: 1st National Fluid Dynamics Conference, American Institute of Aeronautics and Astronautics, 1988.

[25] B. G. van der Wall, J. G. Leishman, On the influence of time-varying flow velocity on unsteady aerodynamics, Journal of The American Helicopter Society 39 (4) (1994) $25-36$.

[26] B. Glaz, L. Liu, P. Friedmann, J. Bain, L. Sankar, A surrogate based approach to reduced-order dynamic stall modeling, in: 51st AIAA/ASME/ASCE/AHS/ASC Structures, Structural Dynamics, and Materials Conference, American Institute of Aeronautics and Astronautics, 2010 .

[27] A. I. Jose, J. G. Leishman, J. D. Baeder, Unsteady aerodynamic modeling with time-varying free-stream mach numbers, Journal of the American Helicopter Society 51 (4) (2006) 299-318. 
[28] K. Gharali, D. A. Johnson, Dynamic stall simulation of a pitching airfoil under unsteady freestream velocity, Journal of Fluids and Structures 42 (2013) $228-244$.

[29] C. Strangfeld, H. Mueller-Vahl, D. Greenblatt, C. Nayeri, C. O. Paschereit, Airfoil subjected to high-amplitude free-stream oscillations: theory and experiments, in: 7th AIAA Theoretical Fluid Mechanics Conference, AIAA Aviation, American Institute of Aeronautics and Astronautics, 2014.

[30] F. Palacios, , M. R. Colonno, A. C. Aranake, A. Campos, S. R. Copeland, T. D. Economon, A. Lonkar, T. W. Lukaczyk, T. W. R. Taylor, J. J. Alonso, Stanford University Unstructured (SU2): An opensource integrated computational environment for multi-physics simulation and design, 51st AIAA Aerospace Sciences Meeting including the New Horizons Forum and Aerospace Exposition.

[31] P. Spalart, S. Allmaras, A one-equation turbulence model for aerodynamic flows, in: 30th Aerospace Sciences Meeting and Exhibit, AIAA, Reno, NV, 1992.

[32] F. Fusi, Robust shape optimization of fixed and morphing rotorcraft airfoils, Ph.D. Thesis, Politecnico di Milano (January 2016).

[33] J. D. J. Anderson, Introduction to flight, McGraw-Hill International Edition, 2005.

[34] G. Kenway, J. R. R. A. Martins, Multipoint high-fidelity aerostructural optimization of a transport aircraft configuration, Journal of Aircraft 51 (1) (2014) 144-160. 
[35] G. Wilke, Applying multi-objective variable-fidelity optimization techniques to industrial scale rotors: Blade designs for cleansky, in: Prooceedings of the European Rotorcraft Forum, Munich, Germany, 2015.

[36] R. L. Bielawa, Rotary wing structural dynamics and aeroelasticity, AIAA, 2006, 2nd Edition.

[37] B. M. Kulfan, J. E. Bussoletti, "Fundamental" Parametric Geometry Representations for Aircraft Component Shapes, in: 11th AIAA/ISSMO Multidisciplinary Analysis and Optimization Conference, Portsmouth, VA, 2006.

[38] M. Drela, Xfoil: An analysis and design system for low reynolds number airfoils., Conference on Low Reynolds Number Airfoil Aerodynamics, University of Notre Dame 54 (1989) 1-12.

[39] M. B. Giles, M. Drela, Viscous-inviscid analysis of transonic and low reynolds number airfoils, AIAA Journal 25 (10) (1987) 1347-1355.

[40] G. Zografakis, G. Barakos, Transition modelling for rotorcraft CFD, in: 34th European Rotorcraft Forum, Liverpool, UK, 2008.

[41] F. Palacios, T. D. Economon, A. C. Aranake, S. R. Copeland, A. K. Lonkar, T. W. Lukaczyk, D. E. Manosalvas, K. R. Naik, A. S. Padron, B. Tracey, A. Variyar, J. J. Alonso, Stanford University Unstructured (SU2): Open-source Analysis and Design Technology for Turbulent Flows, in: 52nd Aerospace Sciences Meeting, 2014.

[42] C. Hirsch, Numerical Computation of Internal and External Flows, second edition Edition, Butterworth-Heinemann, Oxford, 2007. 
[43] N. Srinivas, K. Deb, Multiobjective function optimization using nondominated sorting genetic algorithms, Evolutionary Computation 2 (3) (1994) 221-248.

[44] P. Congedo, C. Corre, J.-M. Martinez, Shape optimization of an airfoil in a BZT flow with multiple-source uncertainties, Computer Methods in Applied Mechanics and Engineering 200 (1-4) (2011) 216-232.

[45] D. S. Lee, J. Periaux, E. Onate, L. F. Gonzalez, N. Qin, Active Transonic Aerofoil Design Optimization Using Robust Multiobjective Evolutionary Algorithms, Journal of Aircraft 48 (3) (2011) 1084-1094.

[46] R. A. E. Mäkinen, J. Periaux, J. Toivanen, Shape design optimization in $2 \mathrm{~d}$ aerodynamics using genetic algorithms on parallel computers, in: Parallel Computational Fluid Dynamics, Elsevier Science, 1995, pp. 395-402.

[47] D. Xiu, G. E. Karniadakis, The Wiener-Askey Polynomial Chaos for Stochastic Differential Equations, SIAM Journal on Scientific Computing 24 (2) (2002) 619-644.

[48] F. Fusi, P. Congedo, A. Guardone, G. Quaranta, Shape optimization under uncertainty of morphing airfoils, Acta Mechanica (2017) 1-22Article in Press. doi:10.1007/s00707-017-2049-3. 